\title{
INTEGRAL view on Cataclysmic Variables and Symbiotic Binaries
}

\author{
Alexander Lutovinov \\ Space Research Institute, Profsoyuznaya str. 84/32, 117997 Moscow, Russia \\ Moscow Institute of Physics and Technology, Dolgoprudny, Moscow Region, 141700, Russia \\ Valery Suleimanov \\ Institute for Astronomy and Astrophysics, University of Tübingen, Sand 1, 72026 \\ Tübingen, Germany \\ Space Research Institute, Profsoyuznaya str. 84/32, 117997 Moscow, Russia \\ Kazan Federal University, Kremlevskaya 18, 420008 Kazan, Russia \\ Gerardo Juan Manuel Luna \\ CONICET-Universidad de Buenos Aires, Instituto de Astronoma y Fsica del Espacio \\ (IAFE), Av. Inte. Güiraldes 2620, C1428ZAA, Buenos Aires, Argentina \\ Universidad de Buenos Aires, Facultad de Ciencias Exactas y Naturales, Buenos Aires, \\ Argentina \\ Universidad Nacional de Hurlingham, Av. Gdor. Vergara 2222, Villa Tesei, Buenos Aires, \\ Argentina.

\section{Sergey Sazonov} \\ Space Research Institute, Profsoyuznaya str. 84/32, 117997 Moscow, Russia \\ Moscow Institute of Physics and Technology, Dolgoprudny, Moscow Region, 141700, Russia \\ Domitilla de Martino \\ INAF - Capodimonte Astronomical Observatory Naples Via Moiariello 16, I-80131 Naples, \\ Italy \\ Lorenzo Ducci \\ Institute for Astronomy and Astrophysics, University of Tübingen, Sand 1, 72026 \\ Tübingen, Germany \\ ISDC Data Center for Astrophysics, Université de Genève, 16 chemin d'Écogia, 1290 \\ Versoix, Switzerland \\ Victor Doroshenko \\ Institute for Astronomy and Astrophysics, University of Tübingen, Sand 1, 72026 \\ Tübingen, Germany \\ Space Research Institute, Profsoyuznaya str. 84/32, 117997 Moscow, Russia \\ Maurizio Falanga \\ International Space Science Institute, Hallerstrasse 6, CH-3012 Bern, Switzerland \\ Email address: aal@iki.rssi.ru (Alexander Lutovinov)
}




\begin{abstract}
Accreting white dwarfs (WDs) constitute a significant fraction of the hard X-ray sources detected by the INTEGRAL observatory. Most of them are magnetic Cataclysmic Variables (CVs) of the intermediate polar (IP) and polar types, but the contribution of the Nova-likes systems and the systems with optically thin boundary layers, Dwarf Novae (DNs) and Symbiotic Binaries (or Symbiotic Stars, SySs) in quiescence is also not negligible. Here we present a short review of the results obtained from the observations of cataclysmic variables and symbiotic binaries by INTEGRAL. The highlight results include the significant increase of the known IP population, determination of the WD mass for a significant fraction of IPs, the establishment of the luminosity function of magnetic CVs, and uncovering origin of the Galactic ridge X-ray emission which appears to largely be associated with hard emission from magnetic CVs.

Keywords: Cataclysmic Variables, Symbiotic Stars, X-ray, White Dwarfs
\end{abstract}

\title{
1. Introduction
}

Cataclysmic variables (CVs) is a broad class of close binary systems with an accreting white dwarf (WD) as a primary (see a detailed review in the book by B. Warner [1]). The virial temperature of protons at the WD surface is about a few tens of keV, so the optically thin accretion flow shocked above the WD surface, inevitably produces an optically thin free-free radiation with the comparable temperatures. Therefore, a significant fraction of CVs appear as hard X-ray sources.

The first model describing this process in the case of the spherical accretion on the WD surface was proposed by [2]. Soon it was recognized that this model is oversimplified and far from the reality. The local shock areas near magnetic poles, which are shaped by the strong magnetic field of the WD, were proposed as the most likely origin of the observed hard X-ray radiation in CVs (see, e.g. [3.) Almost at the same time it was found that the well known CV AM Her was 
detected with the Uhuru observatory as an X-ray source $(3 \mathrm{U} 1809+50)$ [4]. The source also showed a significant optical polarization [5], indicating the presence of a strongly magnetized WD in the system, and thus supported the hypothesis that the observed hard X-ray emission was originating in the hot plasma heated by the shock above the WD surface. For this particular object (AMHer) the spin period is equal to the orbital period, therefore all similar CVs are named synchronous polars (from the polarized optical emission) or AM Her type stars. There is no accretion disc in such systems and the magnetic field strength of the WD $\left(10^{7}-10^{8} \mathrm{G}\right)$ is sufficient to synchronize the spin period with the orbital one. Note that the synchronization is possible only for relatively compact systems, so most polars have relatively short orbital periods $<5 \mathrm{~h}$ (see, e.g., [6]).

The first objects belonging to another type of magnetic CVs, the intermediate polars (IPs), were discovered as X-ray sources in the late of 70th (TV Col [7] and AOPsc [8]). Spin periods of WDs in such systems are much shorter compared to orbital ones, and most of them did not display any optical polarization (see, e.g., 9]). Unlike polars, in IPs systems there was clear evidence for presence of an accretion disc (see details in [1]). The magnetic field strength in these systems is believed to be lower than in polars $\left(<1-3 \times 10^{6} \mathrm{G}\right)$, thus allowing the formation of an accretion disc. It is also not strong enough to synchronize the WD rotation with the orbital period. As a consequence, the accretion disk is truncated at some radius defined as the magnetospheric radius, $R_{\mathrm{m}}$, where the magnetic and accretion pressures balance. Surprisingly, this subclass of the magnetic CVs has been found to be the most numerous among the hard X-ray emitting CVs discovered by INTEGRAL.

A crucial parameter of magnetic CVs, which can be learned from hard X-ray observations, is a mass of the WD. Indeed, the temperature below the shock is defined by a compactness of the WD, i.e., by the ratio of its mass and radius. On the other hand, the relation between mass and radius of the WD is well established (see, e.g. [10]). Therefore, it is possible to estimate the WD mass based on the observed plasma temperature, which in turn can be derived from the approximation of its hard X-ray spectrum with an optically thin emission 
as the bremsstrahlung model (see, e.g., [1] for the first results). Later more detailed models of the so-called post-shock region (PSR) where the observed emission is produced were developed $([12,13,14,15,16])$ and used to estimate the WD masses in magnetic CVs (see, e.g., [17, 18]).

Note, that these early works used a relatively soft $(E<20 \mathrm{keV})$ part of IP Xray spectra measured with the Ginga observatory and $R X T E / \mathrm{PCA}$ instrument. Later, the same approach was applied to observations in hard X-rays with the RXTE/HEXTE, Swift/BAT instruments and with the Suzaku observatory [see, e.g., 19, 20, 21]. A number of articles was dedicated to measure the masses of individual IPs or smaller samples of objects (see, e.g., [22, 23, 24, 25, 26, 27]). Recently, the high sensitivity of the NUSTAR observatory in hard X-rays has been exploited for the same purposes (see, e.g., [28, 29, 30]). The INTEGRAL observatory ([31]) played also an important role in the investigations of CVs. In particular, hard X-ray spectra in many IP systems obtained by the IBIS telescope were used to determine WD masses, starting from the early papers [32, 33].

Obviously, the determination of parameters of individual IPs is possible only for relatively close, bright sources. At the same time an extended hard Xray emission, registered from the Galactic Center and Galactic ridge, can be explained as collective emission of a large number of unresolved CVs. This hypothesis, initially proposed by [34], was later confirmed in number of papers [35, 36, 37. Thus, the study of the Galactic ridge emission is tightly connected with the analysis of individual CVs properties, and the INTEGRAL observatory contributed significantly in this area.

It is important to note that the magnetic CVs are not the only WDs capable of producing hard X-ray emission (see e.g. [38]). For instance, hard X-ray emission from Dwarf Novae (DN) was already discovered at the end of 70s (see, e.g. [39, 40]) during their quiescent states. These results were interpreted as radiation of the optically thin boundary layer between the WD surface and the accretion disc feeding the WD at low mass accretion rates [41, 42]. The system SS Cyg is the best characterized DN in hard X-rays at the present time (see, 
e.g. 43, 44]). In addition to DNs, Novalike systems have been detected in the hard X-rays although some of them are disputed to be magnetic (e.g. IGR J12123-5802 24]; TW Pic [45]) .

Symbiotic Stars (SySs) are a quite small subclass of binary systems, which emit in X-rays due to the accretion onto compact objects. The donor star in such systems is a red giant, and it gives a main contribution in the optical luminosity. A compact object (typically a WD, but there are several systems with neutron stars) accretes the matter from donor wind producing ultraviolet and X-ray emission extending down to the blue optical spectral band (see recent review [46] and references therein). Below we will focus on the systems with WDs as an accretor. The SySs with neutron stars are characterised in a separate review paper in this volume.

The first SySs with accreting WDs were discovered by the Einstein observatory at the beginning of the 80s $47,48,49$. The origin of the X-ray radiation in such systems can be similar to that in CVs although they show also some remarkable difference with respect to them. In particular, there are super-soft sources with permanent thermonuclear burning on the WD surface, like the AG Dra system. Boundary layers between accretion disks and WDs are considered as most probable sources of X-ray radiation in less luminous systems. The hard X-ray emission of RT Cru discovered by INTEGRAL ([50]) was proposed to originate from an optically thin boundary layer.

Here we present a detailed review of the results concerning various types of CVs, obtained with the INTEGRAL observatory. The review includes theoretical explanations discussed in connection with properties of individual sources and their populations such as the luminosity function and contribution to the Galactic ridge emission.

\section{CVs and SySs observed by INTEGRAL}

A significant part of the INTEGRAL observational program is dedicated to surveys of different regions of the Galactic plane and its regular scans with 
the purpose to map the Galaxy, detect new sources and to study in details all detected objects. The main instrument of the observatory for such investigations is the ISGRI detector of the IBIS telescope, ISGRI/IBIS (the Imager on Board the INTEGRAL Satellite / INTEGRAL Soft Gamma Ray Imager, [51]). During more than 15 years INTEGRAL performed the deepest hard X-ray survey of the Galactic plane, which resulted in the discovery and characterization of many new and previously known sources which have been reported in many publications and particularly, the IBIS/ISGRI catalogues (see, e.g., [52, 53, 54]).

These surveys performed with INTEGRAL, besides producing general catalogues, have allowed to study different classes of sources, including the CVs. The first review of CVs, observed with INTEGRAL, was published by [55] and contained 19 known and newly discovered CVs. Most of them were IPs, but a couple of AM Her type systems and one dwarf Nova (SS Cyg) were also included. In a subsequent study, 22 objects were reported [56] and most of them were again IPs. In both works the hard X-ray spectra of the sources were fitted with bremsstrahlung and power-law models. For 11 sources the soft Swift/XRT spectra were also analysed in the follow-up work by [57].

The Galactic plane surveys were extended further to the all-Sky surveys (see, e.g., [52, 54]), which allowed to expand significantly the list of known hard Xray sources. The current version of the general INTEGRAL source catalogu田 contains a few tens of objects, identified as various types of CVs and Symbiotic stars (see Table1). We added some objects only recently identified as CVs, and marked as LMXB a source, which was erroneously identified earlier as CV [58].

\footnotetext{
${ }^{1}$ https://www.isdc.unige.ch/integral/catalog/latest/catalog.html
} 
Table 1: List of CVs and SySs observed by INTEGRAL.

\begin{tabular}{|c|c|c|c|c|c|c|}
\hline $\mathrm{N}$ & Source ID & Name & $\alpha_{2000}$ & $\delta_{2000}$ & Type & Ref \\
\hline 1 & J002324.0+614132 & V1033 Cas* & $00: 22: 57.6$ & $+61: 41: 08$ & IP & 59 \\
\hline 2 & J002848.9+591722 & V709 Cas & $00: 28: 48.9$ & $+59: 17: 22$ & IP & \\
\hline 3 & J005524.0+461211 & V515 And & $00: 55: 24.0$ & $+46: 12: 11$ & IP & 59 \\
\hline 4 & J025604.1+192624 & XY Ari & $02: 56: 04.1$ & $+19: 26: 24$ & IP & \\
\hline 5 & J033111.8+435417 & GK Per & $03: 31: 11.8$ & $+43: 54: 17$ & IP, DN, Nova & \\
\hline 6 & J045707.4+452751 & IGR J04571+4527* & $04: 57: 07.0$ & $+45: 27: 48$ & IP & 60 \\
\hline 7 & J050227.5+244523 & V1062 Tau & $05: 02: 27.5$ & $+24: 45: 23$ & IP & \\
\hline 8 & J051029.3-691012 & IGR J05104-6910* & $05: 10: 29.3$ & $-69: 10: 12$ & DDP? & 61 \\
\hline 9 & J052522.5+241332 & RX J0525.3+2413 & $05: 25: 22.7$ & $+24: 13: 33$ & IP & 25 \\
\hline 10 & J052924.0-324840 & TV Col & $05: 29: 24.0$ & $-32: 48: 40$ & IP & \\
\hline 11 & J053450.5-580139 & TW Pic & $05: 34: 50.5$ & $-58: 01: 39$ & IP? & \\
\hline 12 & J054248.9+605132 & BY Cam & $05: 42: 48.9$ & $+60: 51: 32$ & $\mathrm{AM}$ & \\
\hline 13 & J055807.4+535443 & V405 Aur & 05:58:07.4 & $+53: 54: 43$ & IP & \\
\hline 14 & J061033.6-484426 & V347 Pup & $06: 10: 33.6$ & $-48: 44: 26$ & NL & 62 \\
\hline 15 & J062516.1+733437 & MU Cam & $06: 25: 22.0$ & $+73: 36: 07$ & IP & \\
\hline 16 & J073237.5-133104 & V667 Pup & $07: 32: 37.5$ & $-13: 31: 04$ & IP & \\
\hline 17 & J074623.3-161341 & SWIFT J0746.3-1608 & $07: 46: 23.3$ & $-16: 13: 41$ & IP & 27. \\
\hline 18 & J075117.2+144421 & PQ Gem & $07: 51: 17.4$ & $+14: 44: 25$ & IP & \\
\hline 19 & J080108.2-462244 & 1RXS J080114.6-46232 & 08:01:17.0 & $-46: 23: 27$ & IP & 63 \\
\hline 20 & J083848.9-483125 & IGR J08390-4833* & $08: 38: 49.1$ & $-48: 31: 25$ & IP & 64 \\
\hline 21 & J095750.7-420838 & SWIFT J0958.0-4208 & $09: 57: 50.6$ & $-42: 08: 36$ & IP & \begin{tabular}{|lll}
65 & 63 \\
\end{tabular} \\
\hline 22 & J101050.4-574648 & IGR J10109-5746* & 10:11:03.0 & $-57: 48: 15$ & SyS & 66 \\
\hline 23 & $\mathrm{~J} 114338.2+714121$ & DO Dra & $11: 43: 38.3$ & $+71: 41: 20$ & IP & \\
\hline 24 & J121226.0-580023 & IGR J12123-5802 & $12: 12: 26.2$ & $-58: 00: 21$ & NL or IP? & 24 \\
\hline 25 & J123456.0-643400 & RT Cru & $12: 34: 54.7$ & $-64: 33: 56$ & SyS & \\
\hline 26 & J123816.3-384246 & V1025 Cen & $12: 38: 16.3$ & $-38: 42: 46$ & IP & \\
\hline 27 & J124853.5-624305 & IGR J12489-6243 & $12: 48: 53.5$ & $-62: 43: 05$ & CV? & $\begin{array}{lll}67 & 68 \\
\end{array}$ \\
\hline 28 & J125224.4-291457 & EX Hya & $12: 52: 24.4$ & $-29: 14: 57$ & IP & \\
\hline 29 & J140907.5-451717 & V834 Cen & $14: 09: 07.5$ & $-45: 17: 17$ & $\mathrm{AM}$ & \\
\hline 30 & J140846.0-610754 & IGR J14091-6108* & $14: 08: 46.0$ & $-61: 07: 54$ & IP & 69 \\
\hline 31 & J142507.7-611858 & IGR J14257-6117* & $14: 25: 07.6$ & $-61: 18: 58$ & IP & 65 26 \\
\hline 32 & J145338.0-552223 & IGR J14536-5522* & $14: 53: 41.1$ & $-55: 21: 39$ & $\mathrm{AM}$ & 70 \\
\hline 33 & J150918.8-665100 & IGR J15094-6649* & $15: 09: 26.0$ & $-66: 49: 23$ & IP & 70 \\
\hline 34 & J152915.8-560947 & IGR J15293-5609 & $15: 29: 15.8$ & $-56: 09: 47$ & CV? & 67 68 \\
\hline 35 & J154814.7-452840 & NY Lup & $15: 48: 14.7$ & $-45: 28: 40$ & IP & \\
\hline 36 & J155246.9-502953 & IGR J15529-5029 & $15: 52: 46.9$ & $-50: 29: 53$ & CV? & 71 \\
\hline 37 & $\mathrm{~J} 155930.2+255514$ & $\mathrm{~T} \mathrm{CrB}$ & $15: 59: 30.2$ & $+25: 55: 13$ & SyS & 72 \\
\hline 38 & J161642.0-495700 & IGR J16167-4957* & $16: 16: 37.7$ & $-49: 58: 44$ & IP & 70 \\
\hline 39 & J163553.8-472541 & IGR J16358-4726 & $16: 3$ & $-47: 25: 41$ & SyS & 73 \\
\hline 40 & J165001.2-330658 & IGR J16500-3307* & $16: 49: 55.6$ & $-33: 07: 02$ & IP & 72 \\
\hline 41 & J165443.7-191630 & IGR J16547-1916* & $16: 54: 43.7$ & $-19: 16: 31$ & IP & 74 \\
\hline 42 & J170120.8-430531 & IGR J17014-4306 & $17: 01: 28.2$ & $-43: 06: 12$ & IP & $65]$ \\
\hline 43 & J171236.5-241445 & V2400 Oph & $17: 12: 36.5$ & $-24: 14: 45$ & IP & \\
\hline 44 & J171930.0-410100 & IGR J17195-4100* & $17: 19: 35.9$ & $-41: 00: 54$ & IP & 75 \\
\hline 45 & J172000.0-311600 & IGR J17200-3116 & $17: 20: 05.9$ & $-31: 17: 00$ & SyS? & 68 \\
\hline 46 & J173024.0-060000 & V2731 Oph* & $17: 30: 21.5$ & $-05: 59: 34$ & IP & 76 \\
\hline 47 & J173159.8-191356 & V2487 Oph & $17: 31: 59.8$ & $-19: 13: 56$ & Nova, IP? & 77 \\
\hline 48 & J174017.7-290356 & AX J1740.2-2903 & $17: 40: 16.1$ & $-29: 03: 38$ & IP & 78 \\
\hline 49 & J174026.9-365537 & IGR J17404-3655 & $17: 40: 26.9$ & $-36: 55: 37$ & CV or IP? & \begin{tabular}{|l|l|}
79 & 68 \\
\end{tabular} \\
\hline
\end{tabular}


Table 1 (continue)

\begin{tabular}{|c|c|c|c|c|c|c|}
\hline $\mathrm{N}$ & Source ID & Name & $\alpha_{2000}$ & $\delta_{2000}$ & Type & Ref \\
\hline 50 & J174624.0-213300 & 1RXS J174607.8-21333 & $17: 46: 03.2$ & $-21: 33: 27$ & SyS & 72 \\
\hline 51 & J174955.4-291920 & CXOGBS J174954.5-294335 & $17: 49: 55.4$ & $-29: 19: 20$ & IP & 80 \\
\hline 52 & J175834.6-212322 & IGR J17586-2129* & $17: 58: 34.6$ & $-21: 23: 22$ & SyS? & 68 \\
\hline 53 & $\mathrm{~J} 180035.7+081106$ & V2301 Oph & 18:00:35.8 & $+08: 11: 06$ & $\mathrm{AM}$ & \\
\hline 54 & J180450.6-145450 & IGR J18048-1455 & 18:04:39.0 & $-14: 56: 47$ & IP & 81,82 \\
\hline 55 & J180900.9-274214 & IGR J18088-2741 & 18:09:01.0 & $-27: 42: 14$ & IP? & 83,84 \\
\hline 56 & J181504.0-105132 & IGR J18151-1052 & $18: 15: 03.8$ & $+10: 51: 35$ & IP? & 85, 65. \\
\hline 57 & $\mathrm{~J} 181613.3+495204$ & AM Her & $18: 16: 13.3$ & $+49: 52: 04$ & $\mathrm{AM}$ & \\
\hline 58 & J181722.2-250842 & IGR J18173-2509* & $18: 17: 22.3$ & $-25: 08: 43$ & IP & 86 \\
\hline 59 & J181826.4-235248 & IGR J18184-2352* & $18: 18: 26.4$ & $-23: 52: 48$ & CV? & 54 \\
\hline 60 & J182920.2-121251 & IGR J18293-1213* & $18: 29: 20.2$ & $-12: 12: 51$ & IP? & 87 \\
\hline 61 & J183049.9-123219 & IGR J18308-1232* & $18: 30: 49.9$ & $-12: 32: 19$ & IP & 88 \\
\hline 62 & J1832.3-0840 & AX J1832.3-0840 & $18: 32: 19.3$ & $-08: 40: 30$ & IP & 65 \\
\hline 63 & J185502.2-310948 & V1223 Sgr & $18: 55: 02.2$ & $-31: 09: 48$ & IP & \\
\hline 64 & J190713.6-204554 & V1082 Sgr* & 19:07:13.7 & $-20: 45: 54$ & preCV & 89 \\
\hline 65 & $\mathrm{~J} 192445.6+501414$ & $\mathrm{CH}$ Cyg & $19: 24: 45.6$ & $+50: 14: 14$ & SyS & \\
\hline 66 & J192627.0+132205 & IGR J19267+1325* & $19: 26: 27.0$ & $+13: 22: 05$ & IP & 90 \\
\hline 67 & J194011.5-102525 & V1432 Aql & 19:40:11.5 & $-10: 25: 25$ & $\mathrm{AM}$ & \\
\hline 68 & $\mathrm{~J} 195511.0+004442$ & IGR J19552+0044 & $19: 55: 12.5$ & $+00: 45: 37$ & pre-polar or IP & 24, 91 \\
\hline 69 & $\mathrm{~J} 195814.9+323018$ & V2306 Cyg & $19: 58: 14.9$ & $+32: 30: 18$ & IP & \\
\hline 70 & J201531.4+371116 & RX J2015.6+3711 & $20: 15: 31.4$ & $+37: 11: 17$ & $\mathrm{CV}+$ blazar & 92 \\
\hline 71 & $\mathrm{~J} 210933.8+432046$ & IGR J21095+4322 & 21:09:24.2 & $+43: 19: 36$ & CV? & 93 \\
\hline 72 & $\mathrm{~J} 211352.8+542215$ & 1RXS J211336.1+54222 & $21: 13: 35.4$ & $+54: 22: 33$ & IP & 63 \\
\hline 73 & $\mathrm{~J} 212344.8+421802$ & V2069 Cyg & $21: 23: 44.8$ & $+42: 18: 02$ & IP & \\
\hline 74 & $\mathrm{~J} 213330.0+510531$ & IGR J21335+5105* & $21: 33: 30.0$ & $+51: 05: 31$ & IP & \\
\hline 75 & $\mathrm{~J} 214242.7+433509$ & SS Cyg & $21: 42: 42.8$ & $+43: 35: 10$ & $\mathrm{DN}$ & \\
\hline 76 & J221755.4-082105 & FO Aqr & $22: 17: 55.4$ & $-08: 21: 05$ & IP & \\
\hline 77 & J225518.0-030943 & AO Psc & $22: 55: 18.0$ & $-03: 09: 43$ & IP & \\
\hline 78 & $\mathrm{~J} 232954.3+062811$ & EI Psc & $23: 29: 54.3$ & $+06: 28: 11$ & $\mathrm{DN}$ & \\
\hline
\end{tabular}

Note: * - discovered by INTEGRAL, IP - an intermediate polar, AM - a polar, type AM Her,

DN - a dwarf Nova, NL - a Nova-like star, preCV - a precataclysmic CV, SyS - a symbiotic star, DDP - a double degenerate polar, a secondary star is also WD, LMXB - a low mass X-ray binary. 
In total, the table includes 78 objects: most of them are IPs and candidates (51 sources), seven sources are polars or AMHer type systems, six symbiotic stars, two dwarf Novae (SS Cyg and EI Psc), one pre-cataclysmic variable star (V1082 Sgr), one nova-like star (V347 Pup), and one possible double degenerate polar (IGR J05104-6910). It is possible that the last system is a close binary consisting of two WDs. There are also six candidate CVs which possibly are magnetic systems due to their hard X-ray radiation, and two candidate symbiotic stars. All references associated with the classification or re-classification of individual objects are presented in Table1.

It is important to emphasize that a large fraction of CVs in the Table, namely 21, was either discovered or identified as such with the help of INTEGRAL. These sources are marked with asterisks. Note that some of them had already standard names for variable stars but were only identified as CVs by X-ray observations.

The identification of all hard X-ray sources started immediately after their discoveries by INTEGRAL, and it is obvious that new CVs were also a part of such programs (see, e.g., [70, 59]). Later significant efforts were made for the identifications of the unknown INTEGRAL X-ray sources, and new CVs were also revealed [72, 88, 94, 74, 81, 85, 23, 65]. Definitely, it is not possible to mention all papers devoted to identifications of the INTEGRAL sources, but the works which allowed to establish new CVs are listed in Table1.

The signature feature of IPs are coherent X-ray pulsations with periods ranging from few hundreds to few thousands of seconds. The pulsations reflect the WD spin periods, and for IPs have to be significantly shorter than the orbital period. Therefore, if a candidate CV with a hard X-ray spectrum also displays coherent periodicities, it can be robustly confirmed as a magnetic CV. Most of the newly discovered CVs were identified as magnetic systems using the XMM-Newton observatory that allows long uninterrupted pointings and has high sensitivity in the soft $0.1-10 \mathrm{keV}$ range (see e.g. [95] and references therein), however, INTEGRAL played important role in selection of the candidates. Other X-ray instruments such as Swift/XRT and RXTE also helped 
in establishing the CV nature of a number of INTEGRAL sources (see, e.g., [96, 75, 97, 30]).

Another powerful method to identify CVs is optical spectroscopy. Optical spectra of such objects are characterised by blue continua with many emission lines, mainly corresponding to the Balmer series. Other typical emission features in optical spectra of magnetic CVs are the HeII ( $\lambda 4686)$ line and the emission Bowen fluorescence blend (CIII/NIII spectral lines, seen mainly in the polars). The HeII emission arises from photoionization of material in the accretion disc and magnetically confined accretion flow by X-rays while the latter, the Bowen blend, originates in the irradiated face of the late-type companion star. The common criterion for IPs is an existence of a significant HeII $\lambda 4686$ emission line with the Equivalent Width (EW) of about $10 \AA$. The ratio of EWs between the HeII and $\mathrm{H}_{\beta}$ lines provides a good discriminant for IPs, if $\mathrm{EW}(\mathrm{HeII}) / \mathrm{EW}\left(\mathrm{H}_{\beta}\right)>0.5$ [98. In turn, spectral identification of the optical counterpart as an evolved red giant star (spectral classes KIII - MIII) is a sufficient base to suggest that the given INTEGRAL source is a symbiotic star (see, e.g., [72, 68]).

\section{Models of hard X-ray emission sources in magnetic CVs}

It is commonly accepted now that matter in-falling along the magnetic field lines forms a strong hydro-dynamical shock above the WD surface. The postshock temperature is close to the virial one [2, 3]:

$$
k T_{\mathrm{sh}}=\frac{3}{16} \mu m_{\mathrm{H}} V_{\mathrm{ff}}^{2} \approx 32 M_{1} R_{9}^{-1} \mathrm{keV},
$$

where $\mu \approx 0.62$ is the mean molecular weight of plasma with solar abundances, $m_{\mathrm{H}}$ is the proton mass, $V_{\mathrm{ff}}$ is the pre-shock free-fall velocity of the matter, $M_{1}$ is the WD mass in solar masses, and $R_{9}$ is the WD radius in units of $10^{9} \mathrm{~cm}$. Here we also assume that the kinetic energy of the ions converts equally efficient to the thermal energy of ions and electrons. 
As it was mentioned above, the heated plasma in the PSR settles down as a subsonic flow on the WD surface and cools mainly due to a free-free and cyclotron emissions [3, 99. The plasma velocity and its temperature are decreasing to values typical for the WD surface at the bottom of PSR. The height of the PSR is then determined by the cooling rate of the plasma. This implies that the settling time in the PSR has to be equal to the cooling time of the post-shock plasma. Such post-shock structures are sources of the observed X-ray emission in magnetized CVs.

The observed hard X-ray flux of the PSRs is, therefore, produced by freefree transitions in the hot post-shock plasma. The PSRs are optically thin for emerging free-free radiation, so hard X-ray spectra can be described as an optically thin thermal bremsstrahlung in the simpler case of a plasma with Hydrogen composition. The only free parameter in this case is the temperature, which appears to be an adequate estimate of the post-shock average temperature (see, however, next subsection). This opens the possibility to estimate the WD mass as the relation between WD mass and radius are well known [10. This approach was first suggested and used by Rothschild et al. ([11], see also [100]). It is necessary to note that these estimates can only be correct for relatively weakly magnetized objects, i.e. the IPs. The magnetic field strengths at the WD surface in these objects are $<1-3 \times 10^{6} \mathrm{G}$ [1, 30], which provide the necessary condition for the free-free emission as a dominant cooling mechanism in PSRs in comparison with the cyclotron cooling [99.

The total energy losses due to the cyclotron radiation depend both on the local plasma temperature and magnetic field strength. Therefore, they are most significant just after the shock, which leads to reduction of the maximum PSR temperature and, as a consequence, the observed bremsstrahlung temperature of the X-ray spectra. This is probably one of the reasons why not all polars are hard X-ray sources. However, it is important to note that the free-free cooling can dominate over the cyclotron cooling for luminous polars accreting at high rates, because of the high plasma density [13]. Therefore, such objects can be observed as hard X-ray sources. 
Soon after the paper [11] it was recognized that a single bremsstrahlung spectrum is a rather crude approximation for the emergent spectra of PSRs. 101. suggested to use a number of spectra of optically thin plasma, since the optically thin plasma is multi-temperature. Therefore they used the cooling flow model with the emission measure power defined by the temperature distribution in the flow, i.e. $E M \sim\left(T / T_{\mathrm{sh}}\right)^{\alpha}$. This approach is still used to model hard X-ray spectra of some intermediate polars (see, e.g. 29]). Fully hydro-dynamical PSR models (see below) can also be fitted to this relation (see Table. 2 in 102 for the comparison between different PSR models). Another possible approximation is to assume that the gas pressure is constant in the PSR [103. In this case $\alpha=0.5$, and such a model was also used to study X-ray spectra of IPs (see e.g. [104, 105]).

A more correct PSR model can be obtained using a self-consistent hydrodynamical description. Many works were devoted to this problem, and we can mention here just a few of them ([12, 13, 14]). The simplest model describes the optically thin PSR in the cylindrical geometry cooling only by a thermal radiation without a cyclotron one. We note that more physical and geometrical details should be included into the model to make it fully correct (see, e.g. the dipole geometry importance considered in [15, and the two-temperature plasma approach studied in [13, 16]). Nevertheless, even this simplest model is enough to describe adequately X-ray spectra in the hard energy band. An example of the PSR structure and spectrum of the bright IP V709 Cas, measured with INTEGRAL, are shown in Fig.1

This approach has been implemented in [19] (see also [106]). In the simplest case the model spectrum can be computed as a sum of the local bremsstrahlung spectra. This is adequate to describe only the hard continuum above $\gtrsim 10 \mathrm{keV}$ since the soft part of the observed spectra is dominated by spectral lines and photo-recombination continua. Therefore, the sum of the optically thin plasma spectra over the PSR height rather than multi-temperature bremstrahlung has to be used in this case. In addition, a complex absorption by intervening multiple partial covering neutral material further modifies the soft X-ray spectra of mCVs 

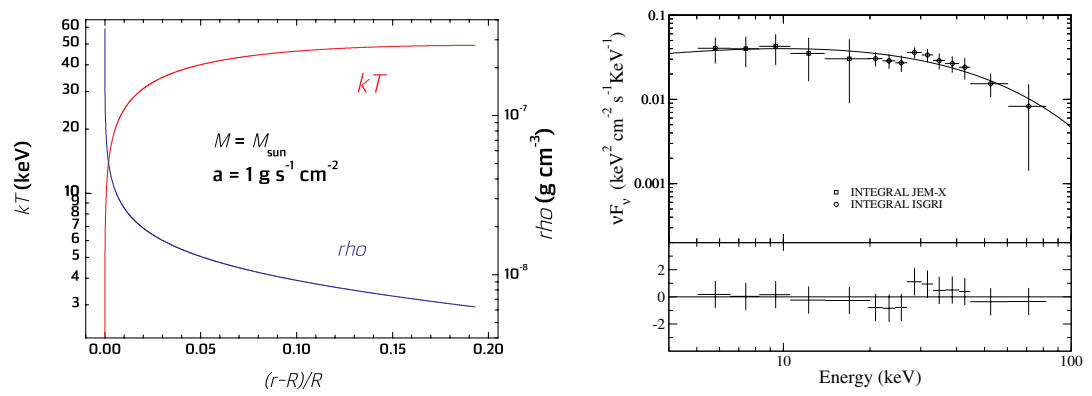

Figure 1: Left: Distributions of temperature (red line) and density (blue line) in the model with $M=M_{\odot}$ and local mass accretion rate $a=1 \mathrm{~g} \mathrm{~s}^{-1} \mathrm{~cm}^{-2}$. Right: The unfolded INTEGRAL JEM-X/ISGRI spectrum of V709 Cas with the best-fit post-shock model. Residuals between the data and model are shown below in units of sigma (from 33 ).

[38. The detection of the fluorescent $\mathrm{Fe} \mathrm{K}_{\alpha}$ line at $6.4 \mathrm{keV}$ indicates the presence of reflection from cold matter, either from the WD or the neutral pre-shock material, which should contribute in the continuum above $10 \mathrm{keV}$. Note that a significant Compton reflection component was found necessary in fitting high S/N spectra of bright IPs as observed by NuSTAR [107, 29.

To avoid the complications associated with the complex nature of the broadband X-ray spectra of IPs for the WD masses determination, it is reasonable, therefore, to restrict the modeling to the hard portion of the observed spectrum $(E>15-20 \mathrm{keV})$ [106, 37, 30].

Another complication, which may make WD mass estimates ambiguous, is a finite size of the magnetosphere which defines the height from which the material falls onto the surface. The free-fall velocity at the shock depends on the magnetospheric radius $R_{\mathrm{m}}$, which is determined as the inner radius of the accretion disc disrupted by the WD magnetic field. Obviously in this case it is possible to determine only a combination of two values: the WD mass $M$ and the relative magnetospheric radius $r_{\mathrm{m}}=R_{\mathrm{m}} / R$. As a consequence, a degeneracy between these two parameters appears. To break this degeneracy and estimate the WD mass, the magnetospheric radius needs to be evaluated independently. So far two approaches have been used to that avail. First, the corotational radius is the natural upper limit for $R_{\mathrm{m}}$ and can be used for the approximate 
estimate of the latter one. Another way is that in some cases the frequency of the break in the power spectrum of the given IPs can be measured, and this frequency is assumed equal to the Kepler frequency at the magnetospheric radius (see 64]). The implementation of this approach is described in [106, 30, and is available as the XSPEC mode2. It is necessary to note, however, that the finite magnetospheric radius affects on the WD mass determination only in the case of the small magnetosphere size $r_{\mathrm{m}}=R_{\mathrm{m}} / R<10$.

The hard X-ray radiation is also observed in non-magnetic CVs, e.g., in some dwarf novae during quiescence, and even Nova-like variables (see Table1). The hard X-ray radiation in these systems originates from the optically thin boundary layer between fast rotating accretion flow and slow rotating WD [41, 42, 108, or even from the optically thin part of the optically thick boundary layer [109]. Spectra of the optically thin boundary layers are also well fitted with the cooling flow model [101]. The generally accepted theory of boundary layers does not yet exist, and we will not describe here any details of different models.

It is important to note, however, that the maximum bremsstrahlung temperature should correspond to the Keplerian velocity at the WD surface, rather than free-fall velocity. As a consequence, the maximum bremsstrahlung temperature has to be by factor of two smaller for the optically thin boundary layer in comparison with the post-shock temperature of a magnetic WD with the same mass. Therefore, the maximum bremsstrahlung temperature of the hard X-ray spectra of non-magnetic CVs can be used for WD mass estimations taking into account the remark above. The hard X-ray radiation of Symbiotic stars is believed to have similar origin as in non-magnetic CVs.

\footnotetext{
${ }^{2}$ https://heasarc.gsfc.nasa.gov/xanadu/xspec/models/ipolar.html
} 


\section{White dwarf masses in magnetized CVs according to the INTEGRAL measurements}

The first estimate of a WD mass with INTEGRAL was made by [110] using the bremsstrahlung temperature obtained from the approximation of the hard X-ray spectrum of the bright IP V1223 Sgr. Subsequently, INTEGRAL observations were used to estimate masses of several WDs using the PSR model and corresponding spectral model grid [19]. In particular, IBIS/ISGRI spectra were used together with the RXTE data to evaluate the WD mass in V2400 Oph [32]; a combination of the IBIS/ISGRI data with JEM-X data was used to determine the WD mass in V709 Cas 33. Later this model grid was used to estimate the WD masses in many IPs (see, e.g., 20, 22, 23]).

The extended INTEGRAL observations of CVs allowed to measure their hard X-ray spectra, which were fitted with the bremsstrahlung and power-law models [55, 56], but these were not used for the WD mass determination. We compiled all published WD mass measurements using the INTEGRAL data in Table2. In line with the corresponding temperatures of the bremsstrahlung emission [55, 56], and for comparison, we also added the WD masses in some IPs recently determined using NuSTAR and Swift/BAT observations and more sophisticated PSR models [30.

The comparison between the WD masses derived from INTEGRAL observations and the values taken from [30] are shown in Fig.2 left panel. Most of the measurements well agree one with another within their $1 \sigma$ uncertainties, with only two IPs, V2400 Oph and IGR J08390-4833, within $3 \sigma$.

As was mentioned above, one of the most straightforward ways to estimate the WD mass in IPs is to use the single bremsstrahlung temperature $k T_{\text {br }}$ obtained from the simple fitting of their hard X-ray spectra. The simplest assumption in this approach is that the bremsstrahlung temperature equals to the maximum temperature of PSR $k T_{\mathrm{sh}}$ (see Eq.11). However, the averaged bremsstrahlung temperature has to be lower than $k T_{\mathrm{sh}}, k T_{\mathrm{br}}=A k T_{\mathrm{sh}}$, where $A<1$. To obtain a real dependence of $k T_{\mathrm{br}}$ on the WD mass we show the 

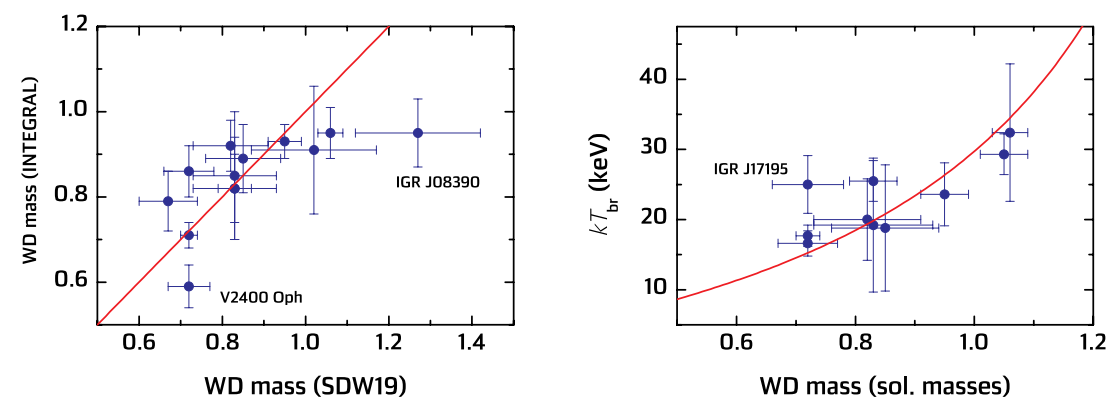

Figure 2: Left: Comparison of the WD masses of the IPs determined using INTEGRAL data with those obtained by [30. Two outlier sources are marked. Right: Dependence of bremsstrahlung temperatures $k T_{\text {br }}$ derived from the hard X-ray spectra of some IPs [56] on the WD mass in these IPs 30. The red curve shows the fit of this dependence with the relation 2.

bremsstrahlung temperatures of IPs, which were found in [56], as a function of WD masses from [30] (Fig. 2, right). Note that we consider here only IPs with a well defined $k T_{\mathrm{br}}$, i.e. with the uncertainty on $T_{\mathrm{br}}$ smaller than $k T_{\mathrm{br}} / 2$. The resulting dependence is well fitted with an approximate relation

$$
k T_{\mathrm{br}}=A k T_{\mathrm{sh}}=\frac{A 32 M_{1}}{1.364 \times\left(1-0.59 M_{1}\right)} \mathrm{keV},
$$

where $A=0.52 \pm 0.03$. Here we used a linear fit for the $M-R$ relation [106. The final expression for calculations of the WD mass using $k T_{\mathrm{br}}$ can be written as

$$
M_{1}=\frac{k T_{\mathrm{br}}}{A \times 23.46+0.59 k T_{\mathrm{br}}} .
$$

Note that this relation is correct for $M_{1}<1.2$ only, because the linear $M-R$ fit is not valid for larger masses. Nevertheless it represents a useful way for the quick WD mass estimates using only hard X-ray data.

Based on the relation (3) we estimated WD masses in three systems, the polar IGR J14536-5522, and the two IPs, IGR J16167-4957 and 2XMMi J180438.7145647) with well determined bremsstrahlung temperatures. Worth mentioning is the estimated mass for the AM Her type system V1432 Aql (see Table2) that is 
Table 2: Observed and derived parameters of magnetic CVs.

\begin{tabular}{|c|c|c|c|c|c|c|c|}
\hline $\mathrm{N}$ & Name & Type & $M_{1}^{a}$ & $k T_{\mathrm{br}}^{b}(\mathrm{keV})$ & $k T_{\mathrm{br}}^{c}(\mathrm{keV})$ & $M_{1}^{e}$ & Ref. \\
\hline 1 & V1033 Cas* & IP & $1.02 \pm 0.15$ & $>14$ & $15.9 \pm 5.1$ & $0.91 \pm 0.15$ & 22 \\
\hline 2 & V709 Cas & IP & $0.83 \pm 0.04$ & $25.5_{-2.7}^{+3.1}$ & $23.3 \pm 2.2$ & $0.82_{-0.25}^{+0.12}$ & 33 \\
\hline 3 & V515 And & IP & $0.67 \pm 0.07$ & & & $0.79 \pm 0.07$ & 23 \\
\hline 4 & GK Per & IP & $0.79 \pm 0.01$ & $43.7_{-23.4}^{+128.8}$ & $28.7 \pm 15.7$ & & \\
\hline 5 & BY Cam & $\mathrm{AM}$ & & $36.7_{-22.7}^{+230.3}$ & $14.8 \pm 5.9$ & $>0.72$ & \\
\hline 6 & MU Cam & IP & $0.67 \pm 0.08$ & $7.0_{-3.8}^{+8.9}$ & $8.1 \pm 4.7$ & & \\
\hline 7 & IGR J08390-4833* & IP & $1.27 \pm 0.15$ & & & $0.95 \pm 0.08$ & 23 \\
\hline 8 & V834 Cen & $\mathrm{AM}$ & & $18.7_{-10.6}^{+61.0}$ & $19.5 \pm 7.8$ & $>0.5$ & \\
\hline 9 & IGR J14536-5522* & IP & & $\begin{array}{l}14.0_{-3.7}^{+5.4} \\
\end{array}$ & $11.1 \pm 4.6$ & $0.68 \pm 0.13$ & \\
\hline 10 & IGR J15094-6649* & IP & $0.85 \pm 0.09$ & $18.8_{-6.5}^{+11.5}$ & $13.8 \pm 5.1$ & $0.89 \pm 0.08$ & 23 \\
\hline 11 & NY Lup & IP & $1.05 \pm 0.04$ & $29.3_{-2.7}^{+3.1}$ & $27.1 \pm 2.2$ & & \\
\hline 12 & IGR J16167-4957* & IP & & $17.3_{-2.7}^{+3.3}$ & $13.2 \pm 2.6$ & $0.77 \pm 0.07$ & \\
\hline 13 & IGR J16500-3307* & IP & $0.82 \pm 0.09$ & $20.0_{-4.7}^{+6.9}$ & & $0.92 \pm 0.06$ & 23 \\
\hline 14 & IGR J16547-1916* & IP & $0.83 \pm 0.10$ & & & $0.85 \pm 0.15$ & 111 \\
\hline 15 & V2400 Oph & IP & $0.72 \pm 0.05$ & $16.6_{-1.7}^{+1.9}$ & $18.6 \pm 1.4$ & $0.59 \pm 0.05$ & 32 \\
\hline 16 & IGR J17195-4100* & IP & $0.72 \pm 0.06$ & $25.0_{-3.6}^{+4.6}$ & $27.0 \pm 4.4$ & $0.86 \pm 0.06$ & 23 \\
\hline 17 & V2731 Oph* & IP & $1.06 \pm 0.03$ & $32.4_{-8.1}^{+11.5}$ & $26.7 \pm 4.4$ & $0.89-1.02$ & 112 \\
\hline 18 & V2487 Oph & IP? & & $55.6_{-24.5}^{+76.4}$ & $25.5 \pm 8.6$ & $>1.0$ & \\
\hline 19 & 2XMMi J180438.7-145647 & IP & & & $\left(23.3_{-5.9}^{+8.84}\right)^{d}$ & $\mathbf{0 . 9 0}+0.13$ & \\
\hline 20 & IGR J18308-1232* & IP & & & & $0.85 \pm 0.06$ & 23 \\
\hline 21 & V1223 Sgr & IP & $0.72 \pm 0.02$ & $17.7_{-1.4}^{+1.6}$ & $18.8 \pm 1.2$ & $0.71 \pm 0.03$ & 110 \\
\hline 22 & V1432 Aql & $\mathrm{AM}$ & & $24.4_{-8.1}^{+15.3}$ & $25.4 \pm 7.0$ & $0.92 \pm 0.18$ & \\
\hline 23 & V2069 Cyg & IP & $0.83 \pm 0.10$ & $19.2_{-6.8}^{+12.3}$ & $35.7 \pm 16.8$ & $0.82 \pm 0.08$ & 23 \\
\hline 24 & IGR J21335+5105* & IP & $0.95 \pm 0.04$ & $23.6_{-4.0}^{+5.0}$ & $23.8 \pm 4.3$ & $0.93 \pm 0.04$ & 22 \\
\hline 25 & FO Aqr & IP & $0.57 \pm 0.03$ & $29.7_{-16.6}^{+70.1}$ & & & \\
\hline
\end{tabular}

Note: * - discovered by INTEGRAL, IP - an intermediate polar, AM - a polar, type AM Her, $a$ - the WD masses from [30], $b-k T_{\mathrm{br}}$ from [56], $c-k T_{\mathrm{br}}$ from [55], $d-k T_{\mathrm{br}}$ taken from [82, $e-$ the WD masses obtained using INTEGRAL observations. The corresponding references are shown in the last column. The boldfaced WD masses given without references were obtained in this work using relation (3), and the bremsstrahlung temperatures measured in 56$] 55,82$.

in remarkable agreement with that derived from $R X T E / \mathrm{PCA}$ data $\left(M_{1} \approx 0.98\right)$ by $[18$.

It is important to note that for some objects the bremsstrahlung temperatures $k T_{\text {br }}$ reported in [56] have large uncertainties, despite the relatively high brightness of these sources (Table 2). A possible reason of this is that their temperatures were determined using the spectra accumulated over a long time 
period (over five years). This implies that the large $k T_{\mathrm{br}}$ uncertainties for the brighter sources may be associated with the intrinsic variability of the spectrum related to mass accretion rate variations, which, in turn, lead to the variations of the magnetospheric radius. For these objects only lower limits on their WD masses are reported in Table2, if any other WD mass determinations are absent. Thus, these systems require additional dedicated investigations to draw conclusions regarding the masses of their WD primaries.

\section{Symbiotic stars}

Symbiotic stars, long-period binary systems where either a white dwarf or a neutron star accretes from the wind of a red giant companion, have been known as X-ray sources since the 70's ([113, 47, 48, 49]). The characteristics of the X-ray emission from symbiotic stars were compiled by [114] using ROSAT data.

Symbiotic stars were classified according to the following scheme (see [114] and following development by [115]):

$\alpha$ : supersoft X-ray sources. Most of their X-ray radiation is emitted below $\approx 0.4 \mathrm{keV}$. They are supposed to be white dwarfs with a quasi-steady shell burning on their surface;

$\beta$ : soft $X$-ray sources. Their X-ray spectra extend up to $\approx 2.4 \mathrm{keV}$ (i.e., the upper bound of the ROSAT energy range). The X-ray photons are likely produced by the collisions of the winds from the white dwarf and the red giant;

$\gamma$ : symbiotic stars with accreting neutron stars (or X-ray symbiotic stars). They have the hard X-ray emission $(E \gtrsim 2.4 \mathrm{keV})$, mostly from the optically thick Comptonizing plasma;

$\delta$ : hard $X$-ray sources with high absorption. They show a strong thermal emission above $E \approx 2.4 \mathrm{keV}$, likely originating in the accretion disc boundary layer; 


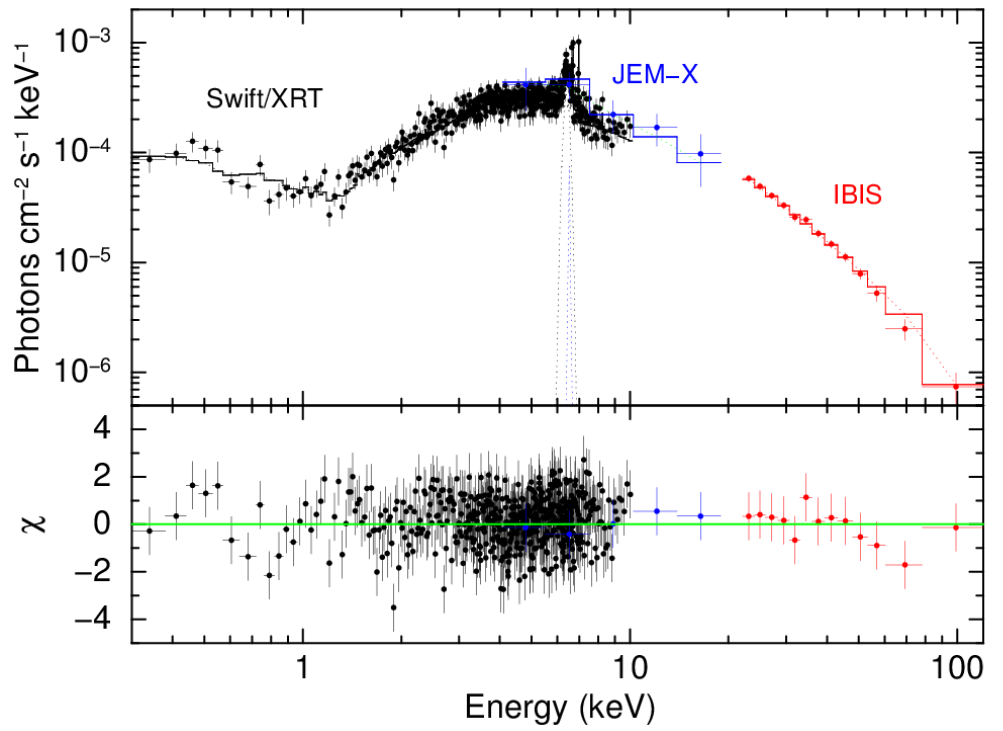

Figure 3: Combined Swift/XRT (black), JEM-X (blue), IBIS/ISGRI (red) unfolded spectra of RT Cru/IGR J12349-6434. The INTEGRAL spectrum was constructed accumulating data from 2003 January 29 and 2014 December 20, while for Swift/XRT the spectrum was built accumulating data from Aug 20, 2005 to Dec 24, 2012 [see [117 for details].

$\beta / \delta$ : symbiotics with soft and hard thermal components. They have properties in common with the subclasses $\beta$ and $\delta$, and the mechanisms producing both spectral components are supposed to be the same of those proposed for $\beta$ and $\delta$ sources.

Only two systems with neutron stars as accreting object were present in the compilation by [114: GX 1+4 and Hen 3-1591. In the modern era of instruments sensitive to energies of more than $10 \mathrm{keV}$ such as INTEGRAL and Swift/BAT, about 10 more similar systems have been discovered. It is believed that their X-ray emission arises from an optically thick Comptonizing plasma with no emission lines [see 116]. These systems are out of scope of the current review and will be reviewed separately in this volume.

The vast majority of symbiotic systems host, however, accreting WDs, making them long-period relatives of CVs. After the aforementioned compilation by [114, [115] reported the first detection of the X-ray emission from eight more 
symbiotic systems using Swift/XRT, thus bringing the total number of known symbiotic systems as X-ray sources to 33 (see also [38]).

Four systems stood out from this sample, RT Cru, T CrB, CD -573057 and $\mathrm{CH}$ Cyg, because of their unprecedented high energy emission. The prototype of them is RT Cru, which was first detected with INTEGRAL/IBIS in 20032004 with the name of IGR J12349-6434 at a $\sim 3$ mCrab level in the 20-60 keV energy band [50. Later, INTEGRAL detected RT Cru again in 2012 with a flux of $\sim 13$ mCrab in the 18-40 keV band [118, and in 2015 at the level of $\sim 6$ $\mathrm{mCrab}$ in the 22-60 keV band [119]. The joint Swift/XRT+INTEGRAL JEM$\mathrm{X} / \mathrm{IBIS} / \mathrm{ISGRI}$ spectrum from RT Cru is shown in Fig. 3 . The system CD -57 3057 was also registered with INTEGRAL during the Crux Galactic arm survey as the IGR J10109-5746 source [34. Moreover, INTEGRAL discovered several new hard X-ray sources, which are considered symbiotic star candidates. The full list of such sources can be found in INTEGRAL catalogues, but here we can mention the most promising objects: IGR J15293-5609 (=CXOU J152929.3561213) [120, IGR J17164-3803 [121] and IGR J17463-2854 [122].

Because of their hard X-ray emission, these objects were dubbed as $\delta$-type $\mathrm{X}$-ray sources in [115. This hard X-ray emission appears to be thermal, and the X-ray spectrum can be modeled with a highly absorbed cooling flow model (see Sect. 3) with maximum temperatures of about $50 \mathrm{keV}$ [123, 124, 125]. Such hard X-ray emission most likely originates in the most internal region of the accretion disk, the boundary layer, which is optically thin to its own radiation if the accretion rate is low, $\dot{M} \lesssim 3 \times 10^{-10} \mathrm{M}_{\odot} \mathrm{yr}^{-1}$ for a $1 \mathrm{M}_{\odot}$ white dwarf [126, 127]. Conversely, the hard X-rays could arise in the accretion column of a magnetic WD, similarly to IPs, although the WD period has not been detected yet in any system [117.

Nevertheless, most of the accretion-powered, hard X-rays emitting, symbiotic systems are too weak to be detected with the current instruments $\left(L_{X} \leq 10^{34}\right.$ $\operatorname{ergs~s}^{-1}$ ). However, Luna et al. [115] found that the amplitude of the flickering in the UV band, with time scales of minutes to hours, (see Fig. 4) is greater in those systems with harder X-ray emission, while sources with low-amplitude 


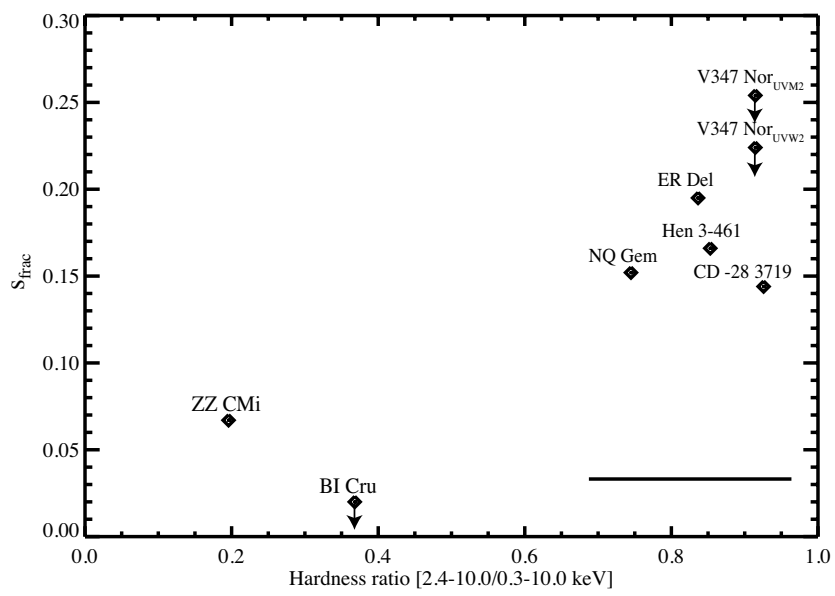

Figure 4: Fractional rms amplitude of flickering $\left(S_{\text {frac }}\right)$ vs. hardness ratio in X-rays. Objects with harder X-ray emission tend to have more intense UV flickering. Downward arrows indicate upper limits. The average error bar is shown at the bottom-right corner [from 115]

UV flickering tend to have relatively little emission above $2 \mathrm{keV}$. This provides a tool to search for accretion-powered systems while hard X-ray observation would be unfeasible long.

As in the case of IPs, the post-shock maximum temperature can be used to determine the WD mass if the X-rays arise in a completely optically thin boundary layer, otherwise, the maximum temperature provides a lower limit for the WD mass. The best-studied systems, RT Cru and T CrB, show strong, long-term variability in their X-ray light curves, which points to changes in the accretion rate and subsequently in the optical depth of the boundary layer.

In the standard accretion disk theory, the total accretion luminosity is divided in equal portions between the Keplerian portion of the accretion disk and the boundary layer. The Keplerian accretion disk radiates mostly in the UV and optical regimes while the boundary layer does it in X-rays. Thus, the ratio of $L_{U V} / L_{X}$ should be equal to one if the boundary layer is completely optically thin. During the high-accretion-rate episodes, such as those in dwarf novae in outburst, or persistent high-accretion regimes as in novae-like, the boundary layer often becomes optically thick to its own radiation, and the peak of 


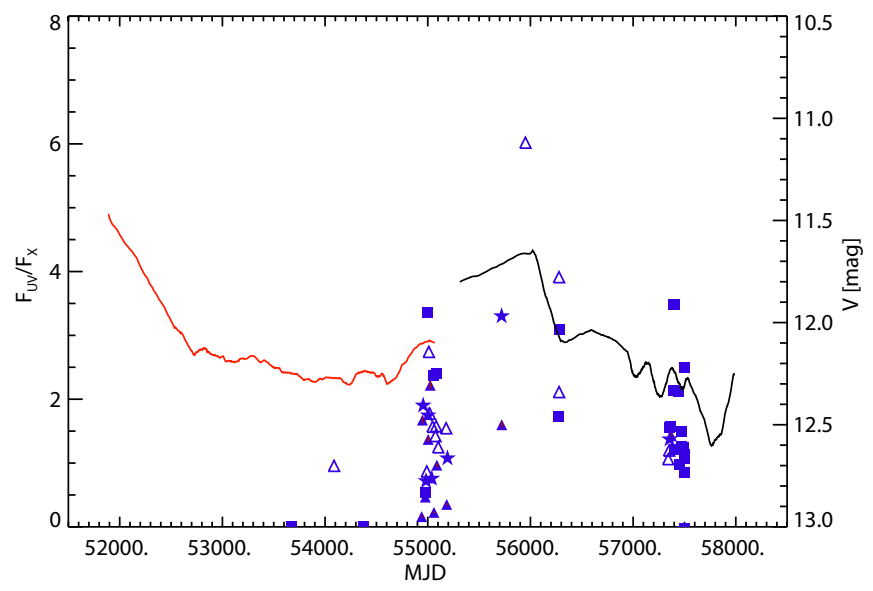

Figure 5: (Left-hand Y-axis) Ratio of reddening-corrected Swift/UVOT magnitudes (U: filled triangle; W1: star; M2: open triangle; W2: square) over unabsorbed X-ray flux in the 0.3-80 $\mathrm{keV}$ band for each Swift observation of RT Cru. (Right-hand Y-axis) Over-plotted are the ASAS (red solid line) and AAVSO (black solid line) V-band light curve, smoothed for clarity [128.

its emission shifts toward longer wavelengths, causing the $L_{U V} / L_{X}$ ratio to be significantly greater than one.

Multiwavelength, UV and X-rays light curves obtained with Swift/XRT and UVOT can be used to trace the optical depth of the boundary layer. 128] used data from the Swift/XRT and UVOT simultaneously and found that, in RT Cru, the $L_{U V} / L_{X}$ is greater than one during the optical maximum while it is closer to one during the optical minimum (Fig. 5). This behavior might be related with the putative orbital period of about 4000 days found in the optical and Swift/BAT light curves.

Another interesting case is that of $\mathrm{T} \mathrm{CrB}$, a symbiotic recurrent nova with a recurrence period of about 80 years. The last thermonuclear outburst happened in 1946. Given that it is the closest recurrent nova, it will certainly produce copious hard-X-rays-to- $\gamma$ rays during the next thermonuclear outburst that could be detected with INTEGRAL.

Since 2014, T CrB entered in what has been called a "super-active" state [129], in which the optical brightness increased by about 1 magnitude, the 15$50 \mathrm{keV}$ flux almost vanished, the UV flux increased by a factor of about 40 
and a new soft X-ray (0.3-1 keV) black body type component emerged. This phenomenology is akin to what is observed during the dwarf nova outbursts, in which the most internal region of the accretion disk changes its optical depth in response to an increase in the accretion rate. However, given the large size of the accretion disk in $\mathrm{T} \mathrm{CrB}$, these changes happen in a far longer time scale.

\section{Luminosity function of CVs}

The determination of the space densities of different classes of CVs in the Galaxy is essential to constrain models of CV formation and evolution. Although fairly large catalogues of CVs, mainly selected in the optical band, have been available for a long time (see [130] and references therein), they were not well suited for determining CV space densities because of the strong and poorly understood selection effects ([131], see also [132]). A breakthrough occurred when the ROSAT observatory conducted an all-sky soft X-ray survey and provided a well-defined, flux limited sample of $\sim 50 \mathrm{CVs}$ (mainly identified among the X-ray sources from the ROSAT Bright Survey at high Galactic latitudes, $|b|>30^{\circ}$ ), which has then been used to estimate the space density of non-magnetic and magnetic CVs [133, 134, 135. Recently, an additional small sub-sample of CVs was identified among the X-ray sources detected in deeper pointed observations with ROSAT, which made it possible to extend the soft X-ray luminosity function of CVs down to $10^{29} \mathrm{erg} \mathrm{s}^{-1}$ [136]. However, the ROSAT survey was limited to energies below $2 \mathrm{keV}$, whereas, as discussed in depth in this review, some classes of CVs such as IPs, emit a large or even dominant fraction of their bolometric luminosity at higher energies. Therefore, in order to conduct a reliable census of such objects it is highly desirable to select them in the hard X-ray band. The first representative sample of CVs selected in a moderately hard X-ray band, 3-20 keV, was obtained by [35] based on the Rossi XTE Slew Survey (XSS) [137]. This sample consisted of 24 known CVs, including 4 non-magnetic ones (dwarf novae), 19 magnetic ones (6 polars and 13 intermediate polars) and 1 symbiotic star. For all but one of these 
objects, distance estimates were available (including several accurate parallax measurements), with most of them located within $500 \mathrm{pc}$ from the Sun. This sample was used to construct the 3-20 keV luminosity function of CVs spanning three decades in luminosity (from $10^{31}$ to $10^{34} \mathrm{erg} \mathrm{s}^{-1}$ ) and to measure the total space density and total X-ray luminosity density (per unit stellar mass) of such objects: $(4.8 \pm 1.6) \times 10^{-7} \mathrm{pc}^{-3}$ and $(2.4 \pm 0.6) \times 10^{27} \mathrm{erg} \mathrm{s}^{-1} M_{\odot}^{-1}(3-20 \mathrm{keV})$, respectively. A relevant study was later performed by [138, where Suzaku, XMM-Newton and ASCA data were used to construct a 2-10 keV luminosity function of dwarf novae and it was shown that this subclass of CVs contributes $\sim 16 \%$ to the total local X-ray luminosity density of CVs.

Afterwards, [36] applied the same approach to the data of INTEGRAL observations and for the first time constructed a sample of CVs selected in a truly hard X-ray band, $17-60 \mathrm{keV}$. To this end, they used the catalogue of sources detected by the IBIS instrument over the whole sky during the first 3.5 years of the INTEGRAL mission [139]. The sample consisted of 17 CVs: 15 intermediate polars (note that the JEM-X telescope aboard INTEGRAL has detected 3 of these objects also in the 5-10 and 10-25 keV energy bands, [140]), one dwarf nova, and one classical nova (V2487 Oph, which also could be a magnetic CV of the IP type [77]). As in previous studies, a serious problem was posed by the highly uncertain distances to some of the studied objects. If the spectral type of the secondary star and the apparent brightness of a CV are known, one can usually attempt to estimate its distance. However, in the case of IPs, the accuracy of this method is significantly affected by the fact that the optical light is mainly produced by the accretion disc rather than the secondary star. Detailed spectroscopic information allows one in some cases to determine the contribution of the light of the companion star to the optical brightness of the binary, but this information was not available for all CVs in the INTEGRAL sample. To overcome these difficulties, the authors took the following novel approach: they measured the correlation between the orbital period and the hard X-ray luminosity for the six CVs with known distances from the sample and used the derived empirical, but physically motivated dependence to predict the 


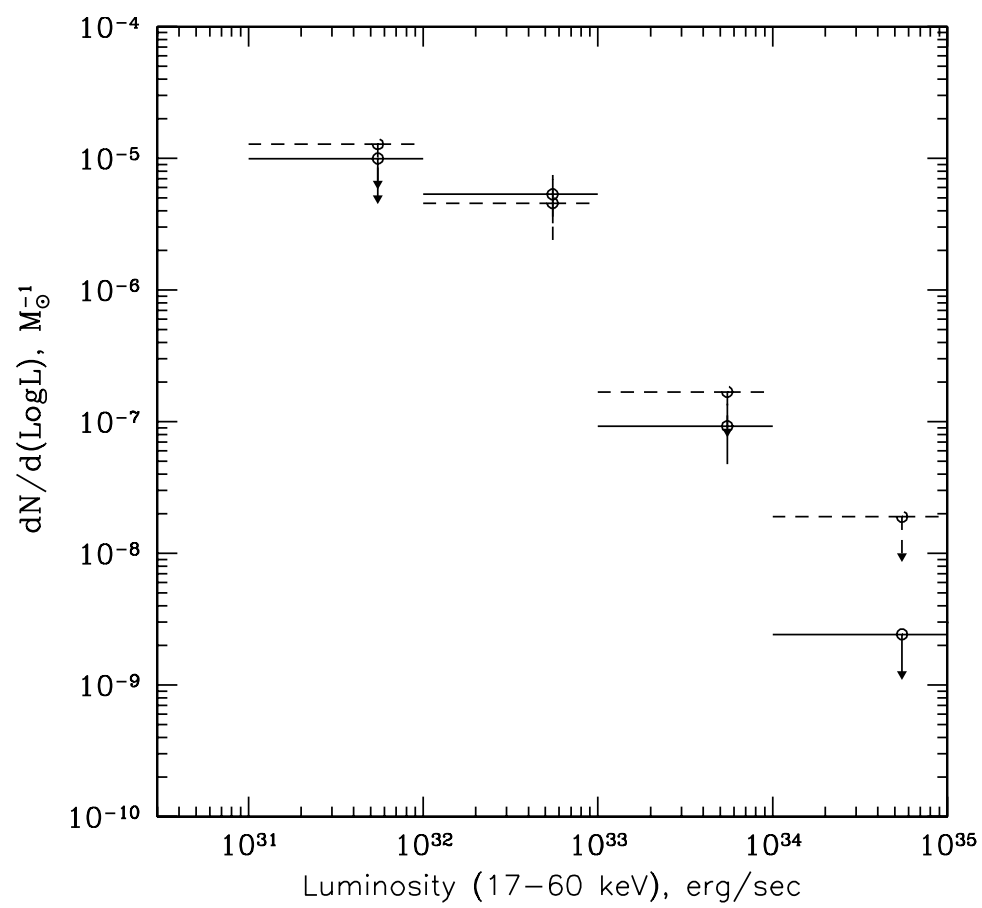

Figure 6: Luminosity function of CVs detected by INTEGRAL over the whole sky (solid crosses). The dashed crosses show the luminosity function obtained excluding the Galactic plane region $\left(|b|<5^{\circ}\right)$, where there remain unidentified INTEGRAL sources. From [36].

hard X-ray luminosities and hence distances for the remaining objects.

The resulting INTEGRAL sample, despite its relatively small size, effectively covered three orders of magnitude in luminosity, $10^{32}-10^{35} \mathrm{erg} \mathrm{s}^{-1}$ (in addition, the absence of sources in the $10^{31}-10^{32} \mathrm{erg} \mathrm{s}^{-1}$ provided an interesting upper limit on the space density of such objects), which made it possible to construct the luminosity function of CVs in the hard X-ray band for the first time (Fig. 6). The integrated space density of CVs with hard X-ray luminosities above $10^{32} \mathrm{erg} \mathrm{s}^{-1}$ proved to be $(1.5 \pm 0.6) \times 10^{-7} \mathrm{pc}^{-3}$ near the Sun, or equivalently $(3.8 \pm 1.5) \times 10^{-6} M_{\odot}^{-1}$ (taking into account the local stellar density $\sim 0.04 M_{\odot} \mathrm{pc}^{-3}$ ). The total X-ray luminosity density of such objects was found to be $(1.3 \pm 0.3) \times 10^{27} \mathrm{erg} \mathrm{s}^{-1} M_{\odot}^{-1}(17-60 \mathrm{keV})$. Finally, it proved possible to estimate the exponential scale height of CVs (primarily IPs) in the Galactic disc at $130_{-46}^{+93} \mathrm{pc}$. 
A similar study has been subsequently carried out using the data of the all-sky hard X-ray survey conducted by the BAT instrument aboard the Swift observatory [141. The authors imposed selection cuts in flux (above $2.5 \times$ $10^{-11} \mathrm{erg} \mathrm{cm}^{-2} \mathrm{~s}^{-1}$ at $\left.14-195 \mathrm{keV}\right)$ and Galactic latitude $\left(|b|>5^{\circ}\right)$ and obtained a sample of $19 \mathrm{CVs}$, including 15 IPs. The latter sub-sample was used to obtain an estimate of the local space density of IPs with hard X-ray (14-195 keV) luminosities above $10^{32} \mathrm{erg} \mathrm{s}^{-1}: 1_{-0.5}^{+1} \times 10^{-7} \mathrm{pc}^{-3}$, in excellent agreement with the INTEGRAL measurement.

The recent second release (DR2) of Gaia parallaxes [142] now offers the opportunity to assess the true space densities and determine more accurate luminosity function. A first work using the shallow flux-limits of the 70-month Swift/BAT sample by [141] and Gaia parallaxes, the IP space density is found to be lower than previously estimated with an upper limit of $<1.3 \times 10^{-7} \mathrm{pc}^{-3}$ although consistent within errors with previous determinations [143. A lower CV space density than predicted by most binary population synthesis models has been recently confirmed by [144] using a volume limited sample of $42 \mathrm{CVs}$ within $150 \mathrm{pc}$. They also find that the fraction of $\mathrm{mCVs}$ is very large $\sim 33 \%$. It is therefore crucial to extend the study to the larger fraction of mCVs detected so far. In this respect it is worth mentioning the successful launch of the Russian-German Spectr-RG mission in summer 2019. The eROSITA and ART-XC telescopes on board of this mission [145, 146] will conduct the most sensitive X-ray survey in the $2-11 \mathrm{keV}$ band to date, and thus promise the discovery of thousands of faint sources pushing the limits of current X-ray luminosity function and assessing the true CV space densities.

\section{Galactic Ridge X-ray Emission}

There are two major large-scale extended diffuse features in the X-ray sky (above $2 \mathrm{keV}$ ): the highly isotropic cosmic X-ray background (CXB) and an emission concentrated towards the Galactic plane - the Galactic ridge X-ray emission (GRXE) [147, 148. While it became clear long ago that the CXB 
is a superposition of numerous active galactic nuclei, the origin of the GRXE remained a puzzle for a long time.

The GRXE energy spectrum contains a number of emission lines of highly ionized heavy elements indicating that the radiation originates in an optically thin plasma with a temperature $\sim 10^{8} \mathrm{~K}$ [149, 150]. The total GRXE luminosity is $\sim(1-2) \times 10^{38} \mathrm{erg} \mathrm{s}^{-1}$ [151]. Historically, two points of view were confronted: (i) the GRXE is the superposition of point-like sources or (ii) it is truly diffuse emission. Each of these hypotheses had its problems. The proponents of the former usually tried to account for the observed GRXE luminosity in terms of the expected contributions from different known classes of faint $\left(L_{X}<10^{34} \mathrm{erg} \mathrm{s}^{-1}\right)$ point sources, but the space densities involved in this estimation were not known accurately enough to draw firm conclusions [152, 153, 154]. The main difficulty with the second scenario was to explain how to keep the hot plasma within the Galaxy or, if it is outflowing, to find the source of energy maintaining this strong wind [150].

In 2006, 155 accomplished a crucial achievement by demonstrating that the GXRE surface brightness, as mapped in the 3-20 keV energy band by RXTE, closely traces the near-infrared (NIR) surface brightness of the Milky Way and hence the distribution of stars throughout the Galaxy. This strongly suggested that the GXRE consists of point-like stellar-type sources. The measured Xray/NIR correlation implied that the cumulative specific X-ray emissivity of these unresolved X-ray sources is $(3.5 \pm 0.5) \times 10^{27} \operatorname{erg~s}^{-1} M_{\odot}^{-1}(3-20 \mathrm{keV})$.

The same team 35 used the RXTE data to construct the luminosity function of CVs (see Sect. 6) and coronally active binary stars (ABs, of RS CVn and other types), in the Solar neighbourhood, which allowed them to determine the integrated 3-20 keV luminosity of such faint $\left(L_{X}<10^{34} \mathrm{erg} \mathrm{s}^{-1}\right)$ X-ray sources per unit stellar mass: $(5.3 \pm 1.5) \times 10^{27} M_{\odot}^{-1} \mathrm{erg} \mathrm{s}^{-1}$. Remarkably, this locally measured quantity proved to be consistent, within the uncertainties, with the X-ray production rate required to explain all of the GRXE (see above). Furthermore, the spatial distribution of $\mathrm{CVs}$ and $\mathrm{ABs}$ is expected to trace the overall distribution of stars in the Galaxy. Therefore, a consistent picture emerged that 


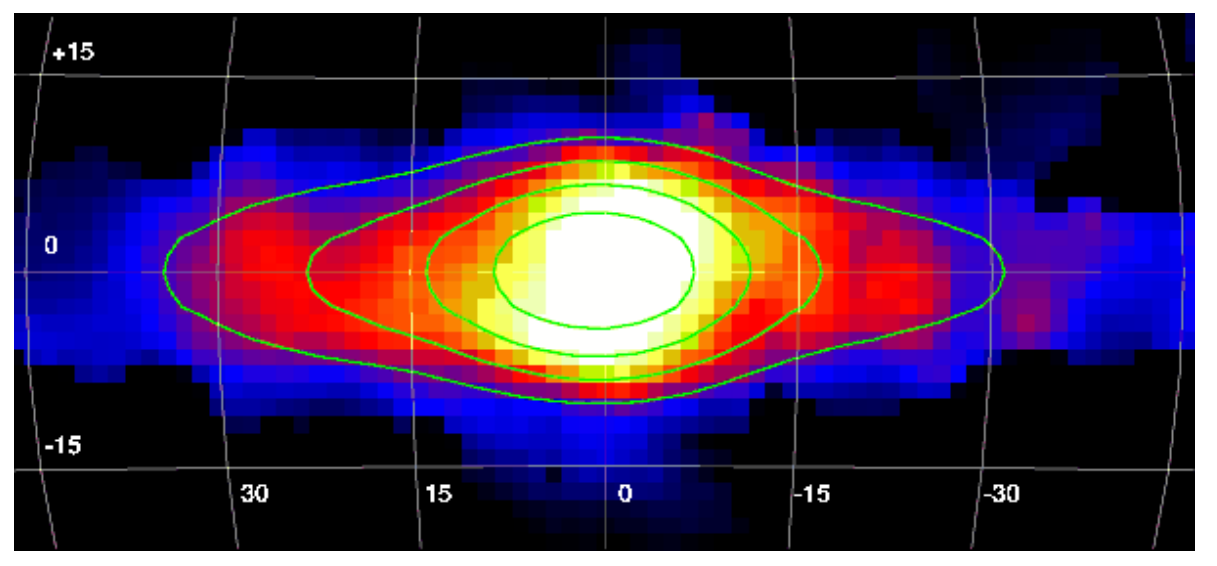

Figure 7: Map of the unresolved Galactic hard X-ray (17-60 keV) emission obtained with INTEGRAL/IBIS, in comparison with the NIR map (contours) obtained by $C O B E /$ DIRBE. From [159.

the bulk of the GRXE is made up by ABs and CVs. This conclusion has later received a spectacular confirmation from the direct resolution of $\sim 80 \%$ of the GRXE at 6-8 keV into discrete sources in ultra-deep Chandra observations of a small region near the Galactic Centre [156, 157.

If the GRXE indeed represents the the sum of the emissions from ABs and CVs, then also the shape of its energy spectrum must be a superposition of representative spectra of these classes of objects. The hard X-ray spectrum has always been a major issue in the study of the GRXE. In particular, if it had a power-law shape, it would imply that non-thermal phenomena, such as cosmic-ray induced emission [158, are involved in the formation of the GRXE. The IBIS telescope aboard INTEGRAL has for the first time combined a large field of view with moderate angular resolution, making it possible to measure the GRXE spectrum by collecting a significant flux of Galactic "diffuse" hard $\mathrm{X}$-rays and separating out the contribution of bright point sources.

Already early studies based on the IBIS observations showed that the GRXE spectrum does not extend into the gamma-ray range from the X-ray band with the same slope [160, 161]. A later, in-depth investigation [159] demonstrated that: (i) the GRXE hard X-ray $(17-60 \mathrm{keV})$ surface brightness is proportional to the NIR surface brightness of the Milky Way (Fig. 7), just like the softer 


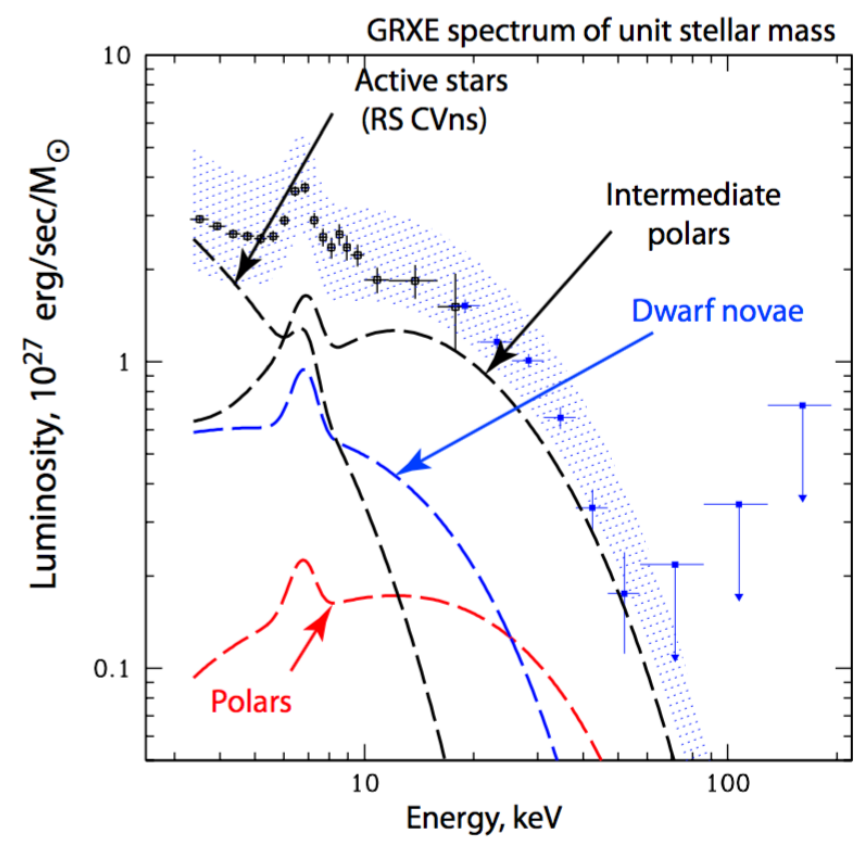

Figure 8: GRXE spectrum measured with $R X T E / \mathrm{PCA}$ (open black squares) and INTE$G R A L /$ IBIS data (filled blue squares), in comparison with typical spectra of the X-ray source classes expected to significantly contribute to the GRXE. These spectra are plotted with normalizations corresponding to their expected relative contributions to the GRXE (derived from the local statistics of faint X-ray sources 35). The shaded region shows the sum of these spectra with the associated uncertainties. From [162].

(3-20 keV) emission, (ii) the inferred GRXE emissivity per unit stellar mass in the $17-60 \mathrm{keV}$ energy band is $(0.9-1.2) \times 10^{27} \mathrm{erg} \mathrm{s}^{-1} M_{\odot}^{-1}$, in excellent agreement with the 17-60 keV luminosity density of CVs in the Solar neighborhood (see the preceding section), and (iii) there is a pronounced cutoff in the GRXE spectrum above 20-30 keV. A comparison of the measured GRXE spectrum with the sum of the expected contributions of different classes of faint X-ray sources, weighted according to their relative contributions to the local X-ray luminosity density, showed an excellent agreement (Fig. 8). The INTEGRAL observations have thus provided strong additional support to the GRXE being the integrated emission of ABs (dominating at energies below $\sim 5 \mathrm{keV}$ ) and CVs (dominating between $\sim 5$ and $\sim 50 \mathrm{keV}$ ), mainly IPs.

The position of the high-energy cutoff in the GRXE spectrum measured by INTEGRAL can be directly linked to the maximal (virial) temperature of the X-ray emitting plasma (see Sect. 3 n near the surface of the accreting magnetic 
WDs composing the GRXE. Based on this idea and the PSR model spectra from [19, [159] estimated the average mass of such objects in the Galaxy to be $\sim 0.5-0.6 M_{\odot}$. A similar value, $0.6-0.7 M_{\odot}$, was obtained in 163 based on Suzaku GRXE observations.

Recently, unresolved extended hard X-ray (20-40 keV) emission has been detected by NUSTAR in the inner few parsecs of the Galaxy [164. Resolved hard X-ray point sources have been further identified in the NuSTAR survey of $0.6 \operatorname{deg}^{2}$ Galactic Center region [165]. As demonstrated by [37, the most natural interpretation of this signal is integrated emission from a large population of IPs in this region. Interestingly, the inferred typical mass of the WDs in these systems, $\sim 0.9 M_{\odot}$, is somewhat higher than that estimated for the whole Galaxy from the INTEGRAL observations of the GRXE (see above), which urges further investigation. However, it is worth noticing that the high mass value is however not so different from that of normal CVs (see [166]). We note in this connection that the average WD mass in nearby bright IPs $\left(0.8-0.9 M_{\odot}\right.$, see [21, 30, 95]) is close to the value found in [37.

\section{Conclusions}

The contribution of the INTEGRAL observatory in the investigations of hard X-ray sky is very significant. The surveys of the Galactic plane and the Galactic center provide a lot of new information about known X-ray sources and allow to discover many new sources. Accreting white dwarfs in close (CVs) and wide (SySs) binary systems were also extensively studied with INTEGRAL. Here we have described the main results obtained with INTEGRAL in this field.

We presented the identified accreting WDs observed with INTEGRAL until November 2019 although many new identifications will further increase this class of hard X-ray emitting galactic sources. The most numerous CVs among them are of the IP type. INTEGRAL discovered 21 new CVs and SySs, including candidates, and the observed X-ray spectra were used for the determination of the WD masses in 18 bright IPs and polars. The WD masses in four sources 
were also estimated in this review for the first time. We also discussed the contribution of the INTEGRAL observatory in the study of symbiotic stars.

The INTEGRAL CV sample has permitted the first measurement of the hard X-ray (17-60 keV) luminosity function of CVs in the Solar neighborhood between $10^{32}$ and $10^{34} \mathrm{erg} \mathrm{s}^{-1}$ (and stringent upper limits on the CV space density at $10^{31}-10^{32}$ and $10^{34}-10^{35} \mathrm{erg} \mathrm{s}^{-1}$ ) and of the total hard X-ray luminosity density of nearby CVs (dominated by IPs): $(1.3 \pm 0.3) \times 10^{27} \mathrm{erg} \mathrm{s}^{-1} M_{\odot}^{-1}$ $(17-60 \mathrm{keV})$. Thanks to the good coverage of the Galactic plane and bulge regions by INTEGRAL observations, it has been demonstrated that the Galactic unresolved hard X-ray emission (from the Galactic Ridge) closely follows the distribution of stars in the Milky Way, with the inferred emissivity per unit stellar mass in the 17-60 keV energy band of $(0.9-1.2) \times 10^{27} \mathrm{erg} \mathrm{s}^{-1} M_{\odot}^{-1}$. The excellent agreement with the aforementioned hard X-ray luminosity density of nearby CVs implies that the GRXE is mainly produced by IPs and other CVs at hard X-ray energies. This conclusion has been further strengthened by the measurement of the GRXE energy spectrum with INTEGRAL, which revealed a cutoff above $20-30 \mathrm{keV}$ as expected from it being the superposition of $\mathrm{CV}$ spectra.

Acknowledgements. This review was supported by several programs and grants. AL, VS and VD acknowledge a support from the Russian Science Foundation, project 19-02-00423. GJML acknowledges financial support from grants ANPCYT-PICT 0478/14, PICT 0901/2017. DdM acknowledges financial support from ASI-INAF contracts I/037/12/0 and .2017-14-H.0 and INAF-PRIN SKA/CTA Presidential Decree 70/2016 and INAF "Sostegno alla ricerca scientifica main streams dell'INAF", Presidential Decree 43/2018. LD acknowledges grant 50 OG 1902. The work of VS was also supported by the DFG grant WE 1312/51-1. 


\section{References}

[1] B. Warner, Cataclysmic Variable Stars, 2003. doi:10.1017/ CB09780511586491.

[2] K. Aizu, X-Ray Emission Region of a White Dwarf with Accretion, Progress of Theoretical Physics 49 (4) (1973) 1184-1194. doi:10.1143/ PTP.49.1184

[3] A. C. Fabian, J. E. Pringle, M. J. Rees, X-ray emission from accretion on to white dwarfs., MNRAS 175 (1976) 43-60. doi:10.1093/mnras/175. 1.43

[4] R. Giacconi, S. Murray, H. Gursky, E. Kellogg, E. Schreier, T. Matilsky, D. Koch, H. Tananbaum, The Third UHURU Catalog of X-Ray Sources, ApJs 27 (1974) 37. doi:10.1086/190288.

[5] S. Tapia, Discovery of a magnetic compact star in the AM Herculis / 3U 1809+50 system., ApJL 212 (1977) L125-L129. doi:10.1086/182390.

[6] A. J. Norton, G. A. Wynn, R. V. Somerscales, The Spin Periods and Magnetic Moments of White Dwarfs in Magnetic Cataclysmic Variables, ApJ 614 (1) (2004) 349-357. arXiv:astro-ph/0406363, doi:10.1086/ 423333 .

[7] P. Charles, J. Thorstensen, S. Bowyer, J. Middleditch, 2A 0526-328: an X-ray-emitting cataclysmic variable., ApJL 231 (1979) L131-L135. doi: 10.1086/183019.

[8] R. E. Griffiths, D. Q. Lamb, M. J. Ward, A. S. Wilson, P. A. Charles, J. Thorstensen, I. M. McHardy, A. Lawrence, The optical identification of H 2252-035 with a cataclysmic variable., MNRAS 193 (1980) 25P-33. doi:10.1093/mnras/193.1.25P.

[9] L. Ferrario, D. de Martino, B. T. Gänsicke, Magnetic White Dwarfs, Space Sci. Rev.191 (1-4) (2015) 111-169. arXiv:1504.08072, doi: $10.1007 / \mathrm{s} 11214-015-0152-0$. 
[10] M. Nauenberg, Analytic Approximations to the Mass-Radius Relation and Energy of Zero-Temperature Stars, ApJ 175 (1972) 417. doi:10.1086/ 151568.

[11] R. E. Rothschild, D. E. Gruber, F. K. Knight, J. L. Matteson, P. L. Nolan, J. H. Swank, S. S. Holt, P. J. Serlemitsos, K. O. Mason, I. R. Tuohy, The X-ray sxpectrum of AM HER from 0.1 to $150 \mathrm{KEV}$., ApJ 250 (1981) 723-732. doi:10.1086/159420.

[12] K. Wu, G. Chanmugam, G. Shaviv, Structure of Steady State Accretion Shocks with Several Cooling Functions: Closed Integral-Form Solution, ApJ 426 (1994) 664. doi:10.1086/174103.

[13] U. Woelk, K. Beuermann, Stationary radiation hydrodynamics of accreting magnetic white dwarfs., A\&A 306 (1996) 232.

[14] M. Cropper, K. Wu, G. Ramsay, A. Kocabiyik, Effects of gravity on the structure of post-shock accretion flows in magnetic cataclysmic variables, MNRAS 306 (3) (1999) 684-690. arXiv:astro-ph/9902355, doi:10. 1046/j.1365-8711.1999.02570.x.

[15] J. B. G. Canalle, C. J. Saxton, K. Wu, M. Cropper, G. Ramsay, Accretion in dipole magnetic fields: flow structure and X-ray emission of accreting white dwarfs, A\&A 440 (1) (2005) 185-198. arXiv:astro-ph/0504061 doi:10.1051/0004-6361:20052706

[16] C. J. Saxton, K. Wu, J. B. G. Canalle, M. Cropper, G. Ramsay, Xray emissions from two-temperature accretion flows within a dipole magnetic funnel, MNRAS 379 (2) (2007) 779-790. arXiv:0705.1331, doi: $10.1111 / \mathrm{j} .1365-2966.2007 .11958 . \mathrm{x}$

[17] M. Cropper, G. Ramsay, K. Wu, White dwarf masses in magnetic cataclysmic variables: multi-temperature FITS to GINGA data, MNRAS 293 (3) (1998) 222-232. doi:10.1046/j.1365-8711.1998.00610.x. 
[18] G. Ramsay, Determining the mass of the accreting white dwarf in magnetic cataclysmic variables using RXTE data, MNRAS 314 (2) (2000) 403-408. arXiv:astro-ph/9912420, doi:10.1046/j.1365-8711.2000.03239.x.

[19] V. Suleimanov, M. Revnivtsev, H. Ritter, RXTE broadband X-ray spectra of intermediate polars and white dwarf mass estimates, A\&A 435 (1) (2005) 191-199. arXiv:astro-ph/0405236, doi:10.1051/0004-6361: 20041283 .

[20] J. Brunschweiger, J. Greiner, M. Ajello, J. Osborne, Intermediate polars in the Swift/BAT survey: spectra and white dwarf masses, A\&A 496 (1) (2009) 121-127. arXiv:0901.3562, doi:10.1051/0004-6361/ 200811285

[21] T. Yuasa, K. Nakazawa, K. Makishima, K. Saitou, M. Ishida, K. Ebisawa, H. Mori, S. Yamada, White dwarf masses in intermediate polars observed with the Suzaku satellite, A\&A 520 (2010) A25. arXiv:1006.5323, doi: 10.1051/0004-6361/201014542.

[22] G. Anzolin, D. de Martino, M. Falanga, K. Mukai, J. M. BonnetBidaud, M. Mouchet, Y. Terada, M. Ishida, Broad-band properties of the hard X-ray cataclysmic variables IGR J00234+6141 and 1RXS J213344.1+510725, A\&A 501 (3) (2009) 1047-1058. arXiv:0905.1080, doi:10.1051/0004-6361/200911816.

[23] F. Bernardini, D. de Martino, M. Falanga, K. Mukai, G. Matt, J. M. Bonnet-Bidaud, N. Masetti, M. Mouchet, Characterization of new hard X-ray cataclysmic variables, A\&A 542 (2012) A22. arXiv:1204.3758, doi:10.1051/0004-6361/201219233.

[24] F. Bernardini, D. de Martino, K. Mukai, M. Falanga, I. Andruchow, J. M. Bonnet-Bidaud, N. Masetti, D. H. G. Buitrago, M. Mouchet, G. Tovmassian, On the nature of the hard X-ray sources SWIFT J1907.3-2050, IGR J12123-5802 and IGR J19552+0044, MNRAS 435 (4) (2013) 2822-2834. arXiv:1308.1230, doi:10.1093/mnras/stt1434 
[25] F. Bernardini, D. de Martino, K. Mukai, G. Israel, M. Falanga, G. Ramsay, N. Masetti, Swift J0525.6+2416 and IGR J04571+4527: two new hard Xray-selected magnetic cataclysmic variables identified with XMM-Newton, MNRAS 453 (3) (2015) 3100-3106. arXiv:1507.06442, doi:10.1093/ mnras/stv1673,

[26] F. Bernardini, D. de Martino, K. Mukai, M. Falanga, IGR J14257-6117, a magnetic accreting white dwarf with a very strong strong X-ray orbital modulation, MNRAS 478 (1) (2018) 1185-1192. arXiv:1804.09899, doi: $10.1093 / \mathrm{mnras} / \mathrm{sty} 1090$.

[27] F. Bernardini, D. de Martino, K. Mukai, M. Falanga, The true nature of Swift J0746.3-1608: a possible Intermediate Polar showing accretion state changes, MNRAS 484 (1) (2019) 101-106. arXiv:1812.09153, doi: $10.1093 / \mathrm{mnras} / \mathrm{sty} 3499$.

[28] A. W. Shaw, C. O. Heinke, K. Mukai, G. R. Sivakoff, J. A. Tomsick, V. Rana, Measuring the masses of intermediate polars with NuSTAR: V709 Cas, NY Lup, and V1223 Sgr, MNRAS 476 (1) (2018) 554-561. arXiv:1801.08508, doi:10.1093/mnras/sty246

[29] Y. Wada, T. Yuasa, K. Nakazawa, K. Makishima, T. Hayashi, M. Ishida, An estimation of the white dwarf mass in the Dwarf Nova GK Persei with NuSTAR observations of two states, MNRAS 474 (2) (2018) 1564-1571. arXiv:1711.01727, doi:10.1093/mnras/stx2880

[30] V. F. Suleimanov, V. Doroshenko, K. Werner, Hard X-ray view on intermediate polars in the Gaia era, MNRAS 482 (2019) 3622-3635. arXiv:1809.05740, doi:10.1093/mnras/sty2952

[31] C. Winkler, T. J. L. Courvoisier, G. Di Cocco, N. Gehrels, A. Giménez, S. Grebenev, W. Hermsen, J. M. Mas-Hesse, F. Lebrun, N. Lund, The INTEGRAL mission, A\&A 411 (2003) L1-L6. doi:10.1051/0004-6361: 20031288 . 
[32] M. G. Revnivtsev, A. A. Lutovinov, B. F. Suleimanov, S. V. Molkov, R. A. Sunyaev, Broadband X-ray Spectrum of the Intermediate Polar V2400 Oph, Astronomy Letters 30 (11) (2004) 772-778. doi:10.1134/1. 1819495 .

[33] M. Falanga, J. M. Bonnet-Bidaud, V. Suleimanov, INTEGRAL broadband X-ray spectrum of the intermediate polar V709 Cassiopeiae, A\&A 444 (2) (2005) 561-564. arXiv:astro-ph/0509489, doi:10.1051/ 0004-6361:20054002.

[34] M. G. Revnivtsev, S. Y. Sazonov, S. V. Molkov, A. A. Lutovinov, E. M. Churazov, R. A. Sunyaev, Hard X-ray survey of the Galactic plane region in Crux: A catalog of sources, Astronomy Letters 32 (3) (2006) 145-148. arXiv:astro-ph/0508155, doi:10.1134/S1063773706030017.

[35] S. Sazonov, M. Revnivtsev, M. Gilfanov, E. Churazov, R. Sunyaev, X-ray luminosity function of faint point sources in the Milky Way, A\&A 450 (1) (2006) 117-128. arXiv:astro-ph/0510049, doi:10.1051/0004-6361: 20054297.

[36] M. Revnivtsev, S. Sazonov, R. Krivonos, H. Ritter, R. Sunyaev, Properties of the Galactic population of cataclysmic variables in hard X-rays, A\&A 489 (3) (2008) 1121-1127. arXiv:0805.2699, doi:10.1051/0004-6361: 200810213

[37] C. J. Hailey, K. Mori, K. Perez, A. M. Canipe, J. Hong, J. A. Tomsick, S. E. Boggs, F. E. Christensen, W. W. Craig, F. Fornasini, J. E. Grindlay, F. A. Harrison, M. Nynka, F. Rahoui, D. Stern, S. Zhang, W. W. Zhang, Evidence for Intermediate Polars as the Origin of the Galactic Center Hard X-ray Emission, ApJ 826 (2) (2016) 160. arXiv:1605.06066, doi: $10.3847 / 0004-637 \mathrm{X} / 826 / 2 / 160$.

[38] K. Mukai, X-Ray Emissions from Accreting White Dwarfs: A Review, PASP 129 (976) (2017) 062001. arXiv:1703.06171, doi:10.1088/ 1538-3873/aa6736. 
[39] J. H. Swank, E. A. Boldt, S. S. Holt, R. E. Rothschild, P. J. Serlemitsos, Hard X-rays form U Geminorum., ApJL 226 (1978) L133-L136. doi: $10.1086 / 182848$.

[40] J. Heise, R. Mewe, A. C. Brinkman, E. H. B. M. Gronenschild, A. J. F. den Boggende, J. Schrijver, D. R. Parsignault, J. E. Grindlay, Detection of both soft and hard X-ray emission from SS Cygni with ANS., A\&A 63 (1978) L1-L3.

[41] J. E. Pringle, G. J. Savonije, X-ray emission from dwarf novae., MNRAS 187 (1979) 777-783. doi:10.1093/mnras/187.4.777.

[42] R. Tylenda, Viscous boundary layer and hard X-rays from dwarf novae., Acta Astronomica 31 (1981) 267-281.

[43] K. E. McGowan, W. C. Priedhorsky, S. P. Trudolyubov, On the Correlated X-Ray and Optical Evolution of SS Cygni, ApJ 601 (2) (2004) 1100-1108. arXiv:astro-ph/0310425, doi:10.1086/380758.

[44] Ş. Balman, M. Revnivtsev, X-ray variations in the inner accretion flow of dwarf novae, A\&A 546 (2012) A112. arXiv:1208.5940, doi:10.1051/ 0004-6361/201219469.

[45] A. J. Norton, A. P. Beardmore, A. Retter, D. A. H. Buckley, The nature of TW Pictoris, MNRAS 312 (2) (2000) 362-370. doi:10.1046/j. 1365-8711.2000.03216.x.

[46] U. Munari, The Symbiotic Stars, arXiv e-prints (2019) arXiv:1909.01389arXiv:1909.01389.

[47] C. M. Anderson, J. P. Cassinelli, W. T. Sanders, X-ray detection of the symbiotic star AG Dra., ApJ 247 (1981) L127-L130. doi:10.1086/ 183605

[48] F. A. Cordova, K. O. Mason, J. E. Nelson, X-ray observations of selected cataclysimic variable stars using the Einstein Observatory., ApJ 245 (1981) 609-617. doi:10.1086/158836. 
[49] D. A. Allen, X-ray observations of symbiotic stars., MNRAS 197 (1981) 739-743. doi:10.1093/mnras/197.3.739.

[50] M. Chernyakova, T. J. L. Courvoisier, J. Rodriguez, A. Lutovinov, INTEGRAL discovery of the new source IGR J12349-6434., The Astronomer's Telegram 519 (2005) 1.

[51] F. Lebrun, J. P. Leray, P. Lavocat, J. Crétolle, M. Arquès, C. Blondel, C. Bonnin, A. Bouère, C. Cara, T. Chaleil, F. Daly, F. Desages, H. Dzitko, B. Horeau, P. Laurent, O. Limousin, F. Mathy, V. Mauguen, F. Meignier, F. Molinié, E. Poindron, M. Rouger, A. Sauvageon, T. Tourrette, ISGRI: The INTEGRAL Soft Gamma-Ray Imager, A\&A 411 (2003) L141-L148. arXiv:astro-ph/0310362, doi:10.1051/0004-6361:20031367.

[52] R. Krivonos, S. Tsygankov, A. Lutovinov, M. Revnivtsev, E. Churazov, R. Sunyaev, INTEGRAL/IBIS nine-year Galactic hard X-ray survey, A\&A 545 (2012) A27. arXiv:1205.3941, doi:10.1051/0004-6361/ 201219617.

[53] A. J. Bird, A. Bazzano, A. Malizia, M. Fiocchi, V. Sguera, L. Bassani, A. B. Hill, P. Ubertini, C. Winkler, The IBIS Soft Gamma-Ray Sky after 1000 Integral Orbits, ApJs 223 (1) (2016) 15. arXiv:1601.06074, doi: $10.3847 / 0067-0049 / 223 / 1 / 15$.

[54] R. A. Krivonos, S. S. Tsygankov, I. A. Mereminskiy, A. A. Lutovinov, S. Y. Sazonov, R. A. Sunyaev, New hard X-ray sources discovered in the ongoing INTEGRAL Galactic plane survey after $14 \mathrm{yr}$ of observations, MNRAS 470 (1) (2017) 512-516. arXiv:1704.03364, doi: $10.1093 / \mathrm{mnras} / \mathrm{stx} 1276$.

[55] E. J. Barlow, C. Knigge, A. J. Bird, A. J Dean, D. J. Clark, A. B. Hill, M. Molina, V. Sguera, 20-100 keV properties of cataclysmic variables detected in the INTEGRAL/IBIS survey, MNRAS 372 (1) (2006) 224-232. arXiv:astro-ph/0607473, doi:10.1111/j.1365-2966.2006.10836.x. 
[56] R. Landi, L. Bassani, A. J. Dean, A. J. Bird, M. Fiocchi, A. Bazzano, J. A. Nousek, J. P. Osborne, INTEGRAL/IBIS and Swift/XRT observations of hard cataclysmic variables, MNRAS 392 (2) (2009) 630-640. arXiv: 0810.1844, doi:10.1111/j.1365-2966.2008.14086.x.

[57] N. Gehrels, G. Chincarini, P. Giommi, K. O. Mason, J. A. Nousek, A. A. Wells, N. E. White, S. D. Barthelmy, D. N. Burrows, L. R. Cominsky, K. C. Hurley, F. E. Marshall, P. Mészáros, P. W. A. Roming, L. Angelini, L. M. Barbier, T. Belloni, S. Campana, P. A. Caraveo, M. M. Chester, O. Citterio, T. L. Cline, M. S. Cropper, J. R. Cummings, A. J. Dean, E. D. Feigelson, E. E. Fenimore, D. A. Frail, A. S. Fruchter, G. P. Garmire, K. Gendreau, G. Ghisellini, J. Greiner, J. E. Hill, S. D. Hunsberger, H. A. Krimm, S. R. Kulkarni, P. Kumar, F. Lebrun, N. M. LloydRonning, C. B. Markwardt, B. J. Mattson, R. F. Mushotzky, J. P. Norris, J. Osborne, B. Paczynski, D. M. Palmer, H. S. Park, A. M. Parsons, J. Paul, M. J. Rees, C. S. Reynolds, J. E. Rhoads, T. P. Sasseen, B. E. Schaefer, A. T. Short, A. P. Smale, I. A. Smith, L. Stella, G. Tagliaferri, T. Takahashi, M. Tashiro, L. K. Townsley, J. Tueller, M. J. L. Turner, M. Vietri, W. Voges, M. J. Ward, R. Willingale, F. M. Zerbi, W. W. Zhang, The Swift Gamma-Ray Burst Mission, ApJ 611 (2) (2004) 10051020. arXiv: astro-ph/0405233, doi:10.1086/422091.

[58] D. de Martino, M. Falanga, J. M. Bonnet-Bidaud, T. Belloni, M. Mouchet, N. Masetti, I. Andruchow, S. A. Cellone, K. Mukai, G. Matt, The intriguing nature of the high-energy gamma ray source XSS J12270-4859, A\&A 515 (2010) A25. arXiv:1002.3740, doi:10.1051/0004-6361/ 200913802

[59] I. F. Bikmaev, M. G. Revnivtsev, R. A. Burenin, R. A. Sunyaev, XSS J00564+4548 and IGR J00234+6141: New cataclysmic variables from the RXTE and INTEGRAL all-sky surveys, Astronomy Letters 32 (9) (2006) 588-593. arXiv:astro-ph/0603715, doi:10.1134/S1063773706090039. 
[60] J. R. Thorstensen, J. Halpern, Optical and X-Ray Studies of 10 X-Rayselected Cataclysmic Binaries, AJ 146 (5) (2013) 107. arXiv:1308.5016, doi: 10.1088/0004-6256/146/5/107.

[61] F. Haberl, G. L. Israel, G. A. Rodriguez Castillo, G. Vasilopoulos, C. Delvaux, A. De Luca, S. Carpano, P. Esposito, G. Novara, R. Salvaterra, A. Tiengo, D. D'Agostino, A. Udalski, EXTraS discovery of two pulsators in the direction of the LMC: a Be/X-ray binary pulsar in the LMC and a candidate double-degenerate polar in the foreground, A\&A 598 (2017) A69. arXiv:1610.00904, doi:10.1051/0004-6361/201629744.

[62] D. A. H. Buckley, D. J. Sullivan, R. A. Remillard, I. R. Tuohy, M. Clark, LB 1800: A Bright Eclipsing Cataclysmic Variable and a Transient X-Ray Source, ApJ 355 (1990) 617. doi:10.1086/168794.

[63] F. Bernardini, D. de Martino, K. Mukai, D. M. Russell, M. Falanga, N. Masetti, C. Ferrigno, G. Israel, Broad-band characteristics of seven new hard X-ray selected cataclysmic variables, MNRAS 470 (4) (2017) 4815-4837. arXiv:1706.04005, doi:10.1093/mnras/stx1494.

[64] M. G. Revnivtsev, A. Y. Kniazev, S. Y. Sazonov, R. A. Burenin, A. Tekola, D. A. H. Buckley, M. L. Pretorius, J. Menzies, W. Lawson, Optical identification of the source IGR J08390-4833 from the INTEGRAL all-sky survey, Astronomy Letters 35 (1) (2009) 33-38. doi: 10.1134/S1063773709010046.

[65] N. Masetti, P. Parisi, E. Palazzi, E. Jiménez-Bailón, V. Chavushyan, V. McBride, A. F. Rojas, L. Steward, L. Bassani, A. Bazzano, A. J. Bird, P. A. Charles, G. Galaz, R. Landi, A. Malizia, E. Mason, D. Minniti, L. Morelli, F. Schiavone, J. B. Stephen, P. Ubertini, Unveiling the nature of INTEGRAL objects through optical spectroscopy. X. A new multi-year, multi-observatory campaign, A\&A 556 (2013) A120. arXiv:1307.2898, doi:10.1051/0004-6361/201322026. 
[66] N. Masetti, L. Bassani, A. J. Dean, P. Ubertini, R. Walter, The symbiotic star CD-57 3057 is the likely counterpart of IGR J10109-5746, The Astronomer's Telegram 715 (2006) 1.

[67] J. A. Tomsick, A. Bodaghee, S. Chaty, J. Rodriguez, F. Rahoui, J. Halpern, E. Kalemci, M. Özbey Arabaci, Localizing INTEGRAL Sources with Chandra: X-Ray and Multi-wavelength Identifications and Energy Spectra, ApJ 754 (2) (2012) 145. arXiv:1206.1071, doi:10. 1088/0004-637X/754/2/145

[68] F. Fortin, S. Chaty, A. Coleiro, J. A. Tomsick, C. H. R. Nitschelm, Spectroscopic identification of INTEGRAL high-energy sources with VLT/ISAAC, A\&A 618 (2018) A150. arXiv:1808.09816, doi:10.1051/ 0004-6361/201731265.

[69] J. A. Tomsick, F. Rahoui, R. Krivonos, M. Clavel, J. Strader, L. Chomiuk, Identifying IGR J14091-6108 as a magnetic CV with a massive white dwarf using X-ray and optical observations, MNRAS 460 (1) (2016) 513-523. arXiv:1604.03562, doi:10.1093/mnras/stw871.

[70] N. Masetti, L. Morelli, E. Palazzi, G. Galaz, L. Bassani, A. Bazzano, A. J. Bird, A. J. Dean, G. L. Israel, R. Landi, A. Malizia, D. Minniti, F. Schiavone, J. B. Stephen, P. Ubertini, R. Walter, Unveiling the nature of INTEGRAL objects through optical spectroscopy. V. Identification and properties of 21 southern hard X-ray sources, A\&A 459 (1) (2006) 21-30. arXiv:astro-ph/0608394, doi:10.1051/0004-6361:20066055.

[71] J. A. Tomsick, S. Chaty, J. Rodriguez, R. Walter, P. Kaaret, Chandra Localizations and Spectra of Integral Sources in the Galactic Plane: The Cycle 9 Sample, ApJ 701 (1) (2009) 811-823. arXiv:0906.2577, doi: 10.1088/0004-637X/701/1/811.

[72] N. Masetti, E. Mason, L. Morelli, S. A. Cellone, V. A. McBride, E. Palazzi, L. Bassani, A. Bazzano, A. J. Bird, P. A. Charles, A. J. Dean, G. Galaz, 
N. Gehrels, R. Landi, A. Malizia, D. Minniti, F. Panessa, G. E. Romero, J. B. Stephen, P. Ubertini, R. Walter, Unveiling the nature of INTEGRAL objects through optical spectroscopy. VI. A multi-observatory identification campaign, A\&A 482 (1) (2008) 113-132. arXiv:0802.0988, doi:10.1051/0004-6361:20079332.

[73] E. Nespoli, J. Fabregat, R. E. Mennickent, IGR J16358-4726 and IGR J16393-4643: two new symbiotic X-ray binaries, The Astronomer's Telegram 1450 (2008) 1.

[74] N. Masetti, P. Parisi, E. Palazzi, E. Jiménez-Bailón, V. Chavushyan, L. Bassani, A. Bazzano, A. J. Bird, A. J. Dean, P. A. Charles, G. Galaz, R. Landi, A. Malizia, E. Mason, V. A. McBride, D. Minniti, L. Morelli, F. Schiavone, J. B. Stephen, P. Ubertini, Unveiling the nature of INTEGRAL objects through optical spectroscopy. VIII. Identification of 44 newly detected hard X-ray sources, A\&A 519 (2010) A96. arXiv: 1006.4513, doi:10.1051/0004-6361/201014852.

[75] O. W. Butters, A. J. Norton, P. Hakala, K. Mukai, E. J. Barlow, RXTE determination of the intermediate polar status of XSS J00564+4548, IGR J17195-4100, and XSS J12270-4859, A\&A 487 (1) (2008) 271-276. arXiv: 0806.0751, doi:10.1051/0004-6361:200809942.

[76] B. T. Gaensicke, T. R. Marsh, A. Edge, P. Rodriguez-Gil, D. Steeghs, S. Araujo-Betancor, E. Harlaftis, O. Giannakis, S. Pyrzas, L. MoralesRueda, A. Aungwerojwit, IGR J17303-0601 is a new intermediate polar, The Astronomer's Telegram 463 (2005) 1.

[77] M. Hernanz, V2487 Oph 1998: a post nova in an intermediate polar, in: European Physical Journal Web of Conferences, Vol. 64 of European Physical Journal Web of Conferences, 2014, p. 07002. doi: 10.1051/epjconf/20136407002.

[78] J. P. Halpern, E. V. Gotthelf, AX J1740.2-2903: Optical Period Confirms 
Intermediate Polar Classification, The Astronomer's Telegram 2681 (2010) 1.

[79] M. Clavel, J. A. Tomsick, J. Hare, R. Krivonos, K. Mori, D. Stern, NuSTAR observations of the unidentified INTEGRAL sources: constraints on the Galactic population of HMXBs, arXiv e-prints (2019) arXiv:1910.02855arXiv:1910.02855,

[80] C. B. Johnson, M. A. P. Torres, R. I. Hynes, P. G. Jonker, C. Heinke, T. Maccarone, C. T. Britt, D. Steeghs, T. Wevers, J. Wu, CXOGBS J174954.5-294335: a new deeply eclipsing intermediate polar, MNRAS 466 (1) (2017) 129-137. arXiv:1612.01612, doi:10.1093/mnras/ stw3063.

[81] D. I. Karasev, A. A. Lutovinov, M. G. Revnivtsev, R. A. Krivonos, Accurate localization and identification of six hard X-ray sources from Chandra and XMM-Newton data, Astronomy Letters 38 (10) (2012) 629-637. arXiv:1209.2945, doi:10.1134/S1063773712100039.

[82] M. J. Middleton, E. M. Cackett, C. Shaw, G. Ramsay, T. P. Roberts, P. J. Wheatley, Identifying a new intermediate polar using XMM-Newton and INTEGRAL, MNRAS 419 (1) (2012) 336-341. arXiv:1108.1105 doi:10.1111/j.1365-2966.2011.19696.x

[83] J. A. Tomsick, R. Krivonos, Q. Wang, A. Bodaghee, S. Chaty, F. Rahoui, J. Rodriguez, F. M. Fornasini, Chandra Observations of Eight Sources Discovered by INTEGRAL, ApJ 816 (1) (2016) 38. arXiv:1512.00044 doi: $10.3847 / 0004-637 \mathrm{X} / 816 / 1 / 38$

[84] F. Rahoui, J. A. Tomsick, R. Krivonos, Identifying four INTEGRAL sources in the Galactic plane via VLT/optical and XMM-Newton/X-ray spectroscopy, MNRAS 465 (2) (2017) 1563-1572. arXiv:1611.02289, doi:10.1093/mnras/stw2830. 
[85] A. A. Lutovinov, R. A. Burenin, M. G. Revnivtsev, I. F. Bikmaev, Optical identification of six hard X-ray sources from the INTEGRAL and SWIFT all-sky surveys, Astronomy Letters 38 (1) (2012) 1-11. doi:10.1134/ S1063773712010045.

[86] E. Nichelli, G. L. Israel, A. Moretti, S. Campana, S. Bernabei, E. Mason, D. Götz, L. Stella, Swift-XRT detection of x-ray pulsations from IGR J18173-2509 and SWIFT J2138.8+5544, The Astronomer's Telegram 2354 (2009) 1.

[87] M. Clavel, J. Tomsick, F. Rahoui, R. Krivonos, Identifying IGR J182931213 and IGR J14091-6108 as magnetic CVs, in: 41st COSPAR Scientific Assembly, Vol. 41, 2016, pp. E1.14-8-16.

[88] N. Masetti, P. Parisi, E. Palazzi, E. Jiménez-Bailón, L. Morelli, V. Chavushyan, E. Mason, V. A. McBride, L. Bassani, A. Bazzano, A. J. Bird, A. J. Dean, G. Galaz, N. Gehrels, R. Landi, A. Malizia, D. Minniti, F. Schiavone, J. B. Stephen, P. Ubertini, Unveiling the nature of INTEGRAL objects through optical spectroscopy. VII. Identification of 20 Galactic and extragalactic hard X-ray sources, A\&A 495 (1) (2009) 121-135. arXiv:0811.4085, doi:10.1051/0004-6361:200811322.

[89] G. Tovmassian, P. Szkody, R. Yarza, M. Kennedy, K2 Study of the Magnetic Precataclysmic Variable V1082 Sagittarius, ApJ 863 (1) (2018) 47. arXiv:1808.03722, doi:10.3847/1538-4357/aad229.

[90] P. A. Evans, A. P. Beardmore, J. P. Osborne, Swift-XRT identification of IGR J19267+1325 as an Intermediate Polar, The Astronomer's Telegram $1669(2008) 1$.

[91] G. Tovmassian, D. González-Buitrago, J. Thorstensen, E. Kotze, H. Breytenbach, A. Schwope, F. Bernardini, S. V. Zharikov, M. S. Hernandez, D. A. H. Buckley, E. de Miguel, F. J. Hambsch, G. Myers, W. Goff, D. Cejudo, D. Starkey, T. Campbell, J. Ulowetz, W. Stein, 
P. Nelson, D. E. Reichart, J. B. Haislip, K. M. Ivarsen, A. P. LaCluyze, J. P. Moore, A. S. Miroshnichenko, IGR J19552+0044: A new asynchronous short period polar. Filling the gap between intermediate and ordinary polars, A\&A 608 (2017) A36. arXiv:1710.02126, doi:10.1051/0004-6361/201731323.

[92] L. Bassani, R. Landi, A. Malizia, J. B. Stephen, A. Bazzano, A. J. Bird, P. Ubertini, The counterpart/s of IGR J20159+3713/SWIFT J2015.9+3715: dissecting a complex region with emission from $\mathrm{keV}$ to TeV, A\&A 561 (2014) A108. arXiv:1310.7837, doi:10.1051/ 0004-6361/201322292.

[93] J. P. Halpern, J. R. Thorstensen, P. Cho, G. Collver, M. Motsoaledi, H. Breytenbach, D. A. H. Buckley, P. A. Woudt, Optical Studies of 15 Hard X-Ray Selected Cataclysmic Binaries, AJ 155 (6) (2018) 247. arXiv: 1804.08532, doi:10.3847/1538-3881/aabfd0.

[94] J. Rodriguez, J. A. Tomsick, S. Chaty, Swift follow-up observations of 17 INTEGRAL sources of uncertain or unknown nature, A\&A 494 (2009) 417-428. arXiv:0811.4707, doi:10.1051/0004-6361:200810773.

[95] D. de Martino, F. Bernardini, K. Mukai, M. Falanga, N. Masetti, Hard Xray Cataclysmic Variables, arXiv e-prints (2019) arXiv:1909.06306arXiv: 1909.06306 doi:10.1016/j.asr.2019.09.006.

[96] J. Brunschweiger, J. Greiner, M. Ajello, J. Osborne, Intermediate polars in the Swift/BAT survey: spectra and white dwarf masses, A\&A 496 (1) (2009) 121-127. arXiv:0901.3562, doi:10.1051/0004-6361/ 200811285 .

[97] O. W. Butters, A. J. Norton, K. Mukai, J. A. Tomsick, RXTE and XMM observations of intermediate polar candidates, A\&A 526 (2011) A77. arXiv:1011.5762, doi:10.1051/0004-6361/201015848, 
[98] J. van Paradijs, F. Verbunt, A comparison of soft x-ray transients and dwarf novae, in: S. E. Woosley (Ed.), American Institute of Physics Conference Series, Vol. 115, 1984, pp. 49-62. doi:10.1063/1.34556.

[99] D. Q. Lamb, A. R. Masters, X and UV radiation from accreting magnetic degenerate dwarfs., ApJL 234 (1979) L117-L122. doi:10.1086/183121.

[100] M. Ishida, Ph.D. thesis, - (Jan 1991).

[101] C. Done, J. P. Osborne, The X-ray spectrum of the dwarf nova SS CYG in quiescence and outburst, MNRAS 288 (3) (1997) 649-664. arXiv: astro-ph/9703036, doi:10.1093/mnras/288.3.649.

[102] M. Falanga, J. M. Bonnet-Bidaud, V. Suleimanov, INTEGRAL broadband X-ray spectrum of the intermediate polar V709 Cassiopeiae, A\&A 444 (2005) 561-564. arXiv:astro-ph/0509489, doi:10.1051/ 0004-6361:20054002

[103] J. Frank, A. King, D. J. Raine, Accretion Power in Astrophysics: Third Edition, 2002.

[104] A. P. Beardmore, J. P. Osborne, C. Hellier, The multi-temperature Xray spectrum of the intermediate polar V1223 Sagittarii, MNRAS 315 (2) (2000) 307-315. doi:10.1046/j.1365-8711.2000.03477.x.

[105] G. J. M. Luna, J. C. Raymond, N. S. Brickhouse, C. W. Mauche, V. Suleimanov, Testing the cooling flow model in the intermediate polar EX Hydrae, A\&A 578 (2015) A15. arXiv:1504.01342, doi:10.1051/ 0004-6361/201525755.

[106] V. Suleimanov, V. Doroshenko, L. Ducci, G. V. Zhukov, K. Werner, GK Persei and EX Hydrae: Intermediate polars with small magnetospheres, A\&A 591 (2016) A35. arXiv:1604.00232, doi:10.1051/0004-6361/ 201628301 . 
[107] K. Mukai, V. Rana, F. Bernardini, D. de Martino, Unambiguous Detection of Reflection in Magnetic Cataclysmic Variables: Joint NuSTAR-XMMNewton Observations of Three Intermediate Polars, ApJL 807 (2) (2015) L30. arXiv:1506.07213, doi:10.1088/2041-8205/807/2/L30

[108] R. Narayan, R. Popham, Hard X-rays from accretion disk boundary layers, Nature 362 (6423) (1993) 820-822. doi:10.1038/362820a0.

[109] J. Patterson, J. C. Raymond, X-ray emission from cataclysmic variables with accretion disks. I. Hard X-rays., ApJ 292 (1985) 535-549. doi: $10.1086 / 163187$.

[110] M. Revnivtsev, A. Lutovinov, V. Suleimanov, R. Sunyaev, V. Zheleznyakov, Broadband X-ray spectrum of intermediate polar V1223 Sgr, A\&A 426 (2004) 253-256. arXiv:astro-ph/0403532, doi:10.1051/0004-6361:20040498

[111] A. A. Lutovinov, R. A. Burenin, M. G. Revnivtsev, V. F. Suleimanov, A. Y. Tkachenko, IGR J16547-1916/1RXS J165443.5-191620 - a new intermediate polar from the INTEGRAL galactic survey, Astronomy Letters 36 (12) (2010) 904-909. arXiv:1011.1129, doi:10.1134/ S1063773710120042,

[112] D. de Martino, G. Matt, K. Mukai, J. M. Bonnet-Bidaud, M. Falanga, B. T. Gänsicke, F. Haberl, T. R. Marsh, M. Mouchet, S. P. Littlefair, V. Dhillon, 1RXS J173021.5-055933: a cataclysmic variable with a fastspinning magnetic white dwarf, A\&A 481 (1) (2008) 149-159. arXiv: 0801.3649, doi:10.1051/0004-6361:20078368.

[113] W. H. G. Lewin, G. R. Ricker, J. E. McClintock, X-Rays from a New Variable Source GX 1+4, ApJ 169 (1971) L17. doi:10.1086/180805

[114] U. Muerset, B. Wolff, S. Jordan, X-ray properties of symbiotic stars. II. Systems with colliding winds., A\&A 319 (1997) 201-210. 
[115] G. J. M. Luna, J. L. Sokoloski, K. Mukai, T. Nelson, Symbiotic stars in Xrays, A\&A 559 (2013) A6. arXiv:1211.6082, doi:10.1051/0004-6361/ 201220792

[116] D. M. Marcu, F. Fürst, K. Pottschmidt, V. Grinberg, S. Müller, J. Wilms, K. A. Postnov, R. H. D. Corbet, C. B. Markwardt, M. Cadolle Bel, The 5 hr Pulse Period and Broadband Spectrum of the Symbiotic X-Ray Binary 3A 1954+319, ApJL 742 (1) (2011) L11. arXiv:1111.1019, doi:10. 1088/2041-8205/742/1/L11.

[117] L. Ducci, V. Doroshenko, V. Suleimanov, M. Nikołajuk, A. Santangelo, C. Ferrigno, RT Crucis: a look into the X-ray emission of a peculiar symbiotic star, A\&A 592 (2016) A58. arXiv:1605.09642, doi:10.1051/ 0004-6361/201628242.

[118] V. Sguera, S. P. Drave, L. Sidoli, M. Fiocchi, J. L. Sokoloski, A. J. Bird, A. Bazzano, L. Bassani, E. Kuulkers, A. Malizia, L. Natalucci, A. Tarana, INTEGRAL detection of the symbiotic star RT Cru during a bright hard X-ray state, The Astronomer's Telegram 3887 (2012) 1.

[119] V. Sguera, A. J. Bird, L. Sidoli, The symbiotic star RT Cru entered a new hard X-ray state as detected by INTEGRAL, The Astronomer's Telegram 8448 (2015) 1.

[120] J. A. Tomsick, A. Bodaghee, S. Chaty, J. Rodriguez, F. Rahoui, J. Halpern, E. Kalemci, M. Özbey Arabaci, Localizing INTEGRAL Sources with Chandra: X-Ray and Multi-wavelength Identifications and Energy Spectra, ApJ 754 (2) (2012) 145. arXiv:1206.1071, doi:10. 1088/0004-637X/754/2/145

[121] F. Rahoui, J. A. Tomsick, R. Krivonos, Identifying four INTEGRAL sources in the Galactic plane via VLT/optical and XMM-Newton/X-ray spectroscopy, MNRAS 465 (2) (2017) 1563-1572. arXiv:1611.02289, doi:10.1093/mnras/stw2830. 
[122] D. I. Karasev, S. S. Tsygankov, A. A. Lutovinov, IGR J17463-2854, a possible symbiotic binary system in the galactic center region, Astronomy Letters 41 (8) (2015) 394-406. arXiv:1507.06302, doi:10.1134/ S1063773715080022.

[123] R. K. Smith, R. Mushotzky, K. Mukai, T. Kallman, C. B. Markwardt, J. Tueller, The Symbiotic System SS73 17 Seen with Suzaku, PASJ 60 (2008) S43. arXiv:0708.3833, doi:10.1093/pasj/60.sp1.S43.

[124] G. J. M. Luna, J. L. Sokoloski, The Nature of the Hard X-Ray-Emitting Symbiotic Star RT Cru, ApJ 671 (1) (2007) 741-747. arXiv:0708.2576 doi: $10.1086 / 522576$

[125] R. N. C. Eze, G. J. M. Luna, R. K. Smith, High-Resolution X-ray Spectra of the Symbiotic Star SS73 17, ApJ 709 (2) (2010) 816-822. arXiv: 0912.2766 doi:10.1088/0004-637X/709/2/816

[126] R. Popham, R. Narayan, Accretion Disk Boundary Layers in Cataclysmic Variables. I. Optically Thick Boundary Layers, ApJ 442 (1995) 337. doi : 10.1086/175444.

[127] V. Suleimanov, M. Hertfelder, K. Werner, W. Kley, Modeling the EUV spectra of optically thick boundary layers of dwarf novae in outburst, A\&A 571 (2014) A55. arXiv:1408.4369, doi:10.1051/0004-6361/ 201423724.

[128] G. J. M. Luna, K. Mukai, J. L. Sokoloski, A. B. Lucy, G. Cusumano, A. Segreto, M. Jaque Arancibia, N. E. Nuñez, R. E. Puebla, T. Nelson, F. Walter, X-ray, UV, and optical observations of the accretion disk and boundary layer in the symbiotic star RT Crucis, A\&A 616 (2018) A53. arXiv:1801.02492, doi:10.1051/0004-6361/201832592

[129] U. Munari, S. Dallaporta, G. Cherini, The 2015 super-active state of recurrent nova $\mathrm{T} \mathrm{CrB}$ and the long term evolution after the 1946 outburst, 
New Astronomy 47 (2016) 7-15. arXiv:1602.07470, doi:10.1016/j. newast.2016.01.002

[130] H. Ritter, U. Kolb, Catalogue of cataclysmic binaries, low-mass X-ray binaries and related objects (Seventh edition), A\&A 404 (2003) 301-303. arXiv:astro-ph/0301444, doi:10.1051/0004-6361:20030330.

[131] J. Patterson, The evolution of cataclysmic and low-mass X-ray binaries., ApJs 54 (1984) 443-493. doi:10.1086/190940.

[132] J. Goliasch, L. Nelson, Population Synthesis of Cataclysmic Variables. I. Inclusion of Detailed Nuclear Evolution, ApJ 809 (1) (2015) 80. arXiv: 1607.06217, doi:10.1088/0004-637X/809/1/80.

[133] A. D. Schwope, H. Brunner, D. Buckley, J. Greiner, K. v. d. Heyden, S. Neizvestny, S. Potter, R. Schwarz, The census of cataclysmic variables in the ROSAT Bright Survey, A\&A 396 (2002) 895-910. arXiv: astro-ph/0210059, doi:10.1051/0004-6361:20021386.

[134] M. L. Pretorius, C. Knigge, The space density and X-ray luminosity function of non-magnetic cataclysmic variables, MNRAS 419 (2) (2012) 14421454. arXiv:1109.3162, doi:10.1111/j.1365-2966.2011.19801.x.

[135] M. L. Pretorius, C. Knigge, A. D. Schwope, The space density of magnetic cataclysmic variables, MNRAS 432 (1) (2013) 570-583. arXiv:1303.4270, doi: $10.1093 / \mathrm{mnras} / \mathrm{stt} 499$.

[136] R. A. Burenin, M. G. Revnivtsev, A. Y. Tkachenko, V. S. Vorob'ev, A. N. Semena, A. V. Meshcheryakov, S. N. Dodonov, M. V. Eselevich, M. N. Pavlinsky, Sample of cataclysmic variables detected in the 400d X-ray survey, Astronomy Letters 42 (4) (2016) 240-250. arXiv:1701.05240, doi:10.1134/S1063773716040022.

[137] M. Revnivtsev, S. Sazonov, K. Jahoda, M. Gilfanov, RXTE all-sky slew survey. Catalog of X-ray sources at b $>10^{\circ}$, A\&A 418 (2004) 927-936. arXiv:astro-ph/0402414, doi:10.1051/0004-6361:20035798, 
[138] K. Byckling, K. Mukai, J. R. Thorstensen, J. P. Osborne, Deriving an Xray luminosity function of dwarf novae based on parallax measurements, MNRAS 408 (4) (2010) 2298-2311. arXiv:1006.5932, doi:10.1111/j. 1365-2966.2010.17276.x.

[139] R. Krivonos, M. Revnivtsev, A. Lutovinov, S. Sazonov, E. Churazov, R. Sunyaev, INTEGRAL/IBIS all-sky survey in hard X-rays, A\&A 475 (2) (2007) 775-784. arXiv:astro-ph/0701836, doi:10.1051/0004-6361: 20077191.

[140] S. A. Grebenev, I. A. Mereminskiy, JEM-X/INTEGRAL X-ray survey of the galactic center region, Astronomy Letters 41 (12) (2015) 765-784. doi:10.1134/S1063773715120038

[141] M. L. Pretorius, K. Mukai, Constraints on the space density of intermediate polars from the Swift-BAT survey, MNRAS 442 (3) (2014) 2580-2585. arXiv:1405.3908, doi:10.1093/mnras/stu990.

[142] Gaia Collaboration, Gaia Data Release 2. Summary of the contents and survey properties, A\&A 616 (2018) A1. arXiv:1804.09365, doi: 10.1051/0004-6361/201833051.

[143] A. D. Schwope, Exploring the space density of X-ray selected cataclysmic variables, A\&A 619 (2018) A62. arXiv:1808.08144, doi:10. $1051 / 0004-6361 / 201833723$

[144] A. F. Pala, B. T. Gänsicke, E. Breedt, C. Knigge, J. J. Hermes, N. P. Gentile Fusillo, M. A. Hollands, T. Naylor, I. Pelisoli, S. Toonen, A. Aungwerojwit, E. Cukanovaite, E. Dennihy, C. J. Manser, M. L. Pretorius, S. Scaringi, M. R. Schreiber, O. Toloza, The Space Density of Cataclysmic Variables from Gaia DR2, arXiv e-prints (2019) arXiv:1907.13152arXiv: 1907.13152

[145] A. Merloni, P. Predehl, W. Becker, H. Böhringer, T. Boller, H. Brunner, M. Brusa, K. Dennerl, M. Freyberg, P. Friedrich, A. Georgakakis, 
F. Haberl, G. Hasinger, N. Meidinger, J. Mohr, K. Nandra, A. Rau, T. H.

Reiprich, J. Robrade, M. Salvato, A. Santangelo, M. Sasaki, A. Schwope, J. Wilms, t. German eROSITA Consortium, eROSITA Science Book: Mapping the Structure of the Energetic Universe, arXiv e-prints (2012) arXiv:1209.3114arXiv:1209.3114

[146] M. Pavlinsky, V. Levin, V. Akimov, A. Krivchenko, A. Rotin, M. Kuznetsova, I. Lapshov, A. Tkachenko, R. Krivonos, N. Semena, M. Buntov, A. Glushenko, V. Arefiev, A. Yaskovich, S. Grebenev, S. Sazonov, A. Lutovinov, S. Molkov, D. Serbinov, M. Kudelin, T. Drozdova, S. Voronkov, R. Sunyaev, E. Churazov, M. Gilfanov, B. Ramsey, S. L. O'Dell, J. Kolodziejczak, V. Zavlin, D. Swartz, ART-XC / SRG overview, in: Proc. SPIE, Vol. 10699 of Society of Photo-Optical Instrumentation Engineers (SPIE) Conference Series, 2018, p. $106991 Y$. doi:10.1117/12.2312053

[147] D. M. Worrall, F. E. Marshall, E. A. Boldt, J. H. Swank, HEAO 1 measurements of the galactic ridge., ApJ 255 (1982) 111-121. doi: $10.1086 / 159809$.

[148] R. S. Warwick, M. J. L. Turner, M. G. Watson, R. Willingale, The galactic ridge observed by Exosat, Nature 317 (6034) (1985) 218-221. doi:10. $1038 / 317218 \mathrm{a} 0$

[149] K. Koyama, K. Makishima, Y. Tanaka, H. Tsunemi, Thermal X-ray emission with intense 6.7-keV iron line from the galactic ridge., PASJ 38 (1986) $121-131$.

[150] Y. Tanaka, ASCA observation of X-ray emission from the Galactic ridge, A\&A 382 (2002) 1052-1060. doi:10.1051/0004-6361:20011678.

[151] A. Valinia, F. E. Marshall, RXTE Measurement of the Diffuse X-Ray Emission from the Galactic Ridge: Implications for the Energetics of the Interstellar Medium, ApJ 505 (1) (1998) 134-147. arXiv:astro-ph/ 9804012, doi:10.1086/306155. 
[152] D. M. Worrall, F. E. Marshall, Stellar contributions to the hard X-ray galactic ridge., ApJ 267 (1983) 691-697. doi:10.1086/160906.

[153] R. Ottmann, J. H. M. M. Schmitt, The contributions of RS CVn systems to the diffuse X-ray background., A\&A 256 (1992) 421-427.

[154] K. Mukai, K. Shiokawa, The EXOSAT Medium Energy (ME) Sample of Dwarf Novae, ApJ 418 (1993) 863. doi:10.1086/173444.

[155] M. Revnivtsev, S. Sazonov, M. Gilfanov, E. Churazov, R. Sunyaev, Origin of the Galactic ridge X-ray emission, A\&A 452 (1) (2006) 169-178. doi: 10.1051/0004-6361:20054268.

[156] M. Revnivtsev, S. Sazonov, E. Churazov, W. Forman, A. Vikhlinin, R. Sunyaev, Discrete sources as the origin of the Galactic X-ray ridge emission, Nature 458 (7242) (2009) 1142-1144. arXiv:0904.4649, doi: 10.1038/nature07946.

[157] M. Revnivtsev, S. Sazonov, W. Forman, E. Churazov, R. Sunyaev, Luminosity function of faint Galactic sources in the Chandra bulge field, MNRAS 414 (1) (2011) 495-499. arXiv:1101.5883, doi:10.1111/j. 1365-2966.2011.18411.x

[158] J. G. Skibo, W. N. Johnson, J. D. Kurfess, R. L. Kinzer, G. Jung, J. E. Grove, W. R. Purcell, M. P. Ulmer, N. Gehrels, J. Tueller, OSSE Observations of the Soft Gamma-Ray Continuum from the Galactic Plane at Longitude 95 degrees, ApJL 483 (2) (1997) L95-L98. arXiv:astro-ph/ 9704207, doi:10.1086/310755.

[159] R. Krivonos, M. Revnivtsev, E. Churazov, S. Sazonov, S. Grebenev, R. Sunyaev, Hard X-ray emission from the Galactic ridge, A\&A 463 (3) (2007) 957-967. arXiv:astro-ph/0605420, doi:10.1051/0004-6361: 20065626 .

[160] F. Lebrun, R. Terrier, A. Bazzano, G. Bélanger, A. Bird, L. Bouchet, A. Dean, M. Del Santo, A. Goldwurm, N. Lund, H. Morand, A. Par- 
mar, J. Paul, J. P. Roques, V. Schönfelder, A. W. Strong, P. Ubertini, R. Walter, C. Winkler, Compact sources as the origin of the soft $\gamma$-ray emission of the Milky Way, Nature 428 (6980) (2004) 293-296. doi:10.1038/nature02407.

[161] R. Terrier, F. Lebrun, G. Bélanger, A. Goldwurm, A. W. Strong, V. Schoenfelder, L. Bouchet, J. P. Roques, A. Parmar, Contribution of Point Sources to the Soft Gamma-Ray Galactic Emission, in: V. Schoenfelder, G. Lichti, C. Winkler (Eds.), 5th INTEGRAL Workshop on the INTEGRAL Universe, Vol. 552 of ESA Special Publication, 2004, p. 513. arXiv:astro-ph/0405207.

[162] M. Revnivtsev, S. Sazonov, R. Sunyaev, Origin of the Galactic Ridge X-Ray Emission, Progress of Theoretical Physics Supplement 169 (2007) 125-130. arXiv:astro-ph/0510050, doi:10.1143/PTPS.169.125.

[163] T. Yuasa, K. Makishima, K. Nakazawa, Broadband Spectral Analysis of the Galactic Ridge X-Ray Emission, ApJ 753 (2) (2012) 129. arXiv: 1205.1574, doi:10.1088/0004-637X/753/2/129

[164] K. Perez, C. J. Hailey, F. E. Bauer, R. A. Krivonos, K. Mori, F. K. Baganoff, N. M. Barrière, S. E. Boggs, F. E. Christensen, W. W. Craig, B. W. Grefenstette, J. E. Grindlay, F. A. Harrison, J. Hong, K. K. Madsen, M. Nynka, D. Stern, J. A. Tomsick, D. R. Wik, S. Zhang, W. W. Zhang, A. Zoglauer, Extended hard-X-ray emission in the inner few parsecs of the Galaxy, Nature 520 (7549) (2015) 646-649. doi:10.1038/nature14353.

[165] J. Hong, K. Mori, C. J. Hailey, M. Nynka, S. Zhang, E. Gotthelf, F. M. Fornasini, R. Krivonos, F. Bauer, K. Perez, J. A. Tomsick, A. Bodaghee, J.-L. Chiu, M. Clavel, D. Stern, J. E. Grindlay, D. M. Alexander, T. Aramaki, F. K. Baganoff, D. Barret, N. Barrière, S. E. Boggs, A. M. Canipe, F. E. Christensen, W. W. Craig, M. A. Desai, K. Forster, P. Giommi, B. W. Grefenstette, F. A. Harrison, D. Hong, A. Hornstrup, T. Kitaguchi, J. E. Koglin, K. K. Madsen, P. H. Mao, H. Miyasaka, M. Perri, M. J. 
Pivovaroff, S. Puccetti, V. Rana, N. J. Westergaard, W. W. Zhang, A. Zoglauer, NuSTAR Hard X-Ray Survey of the Galactic Center Region. II. X-Ray Point Sources, ApJ 825 (2) (2016) 132. arXiv:1605.03882, doi: $10.3847 / 0004-637 X / 825 / 2 / 132$.

[166] M. Zorotovic, M. R. Schreiber, B. T. Gänsicke, Post common envelope binaries from SDSS. XI. The white dwarf mass distributions of CVs and preCVs, A\&A 536 (2011) A42. arXiv:1108.4600, doi:10.1051/0004-6361/ 201116626. 